\title{
1 Dynamics of Spaetzle morphogen shuttling in the Drosophila 2 embryo shapes pattern
}

4 Neta Rahimi*, Inna Averbukh*, Shari Carmon, Eyal D. Schejter, Naama Barkai\& and

5 Ben-Zion Shilo\&\#

6 Department of Molecular Genetics, Weizmann Institute of Science, Rehovot 76100,

7 Israel

$8 *$ Equal contribution

9 \& Corresponding authors

10 \# Lead contact

\section{Abstract}

14 Establishment of morphogen gradients in the early Drosophila embryo is challenged by a

15 diffusible extracellular milieu, and rapid nuclear divisions that occur at the same time. To

16 understand how a sharp gradient is formed within this dynamic environment, we followed the

17 generation of graded nuclear Dorsal (D1) protein, the hallmark of pattern formation along the

18 dorso-ventral axis, in live embryos. We show that a sharp gradient is formed through

19 extracellular, diffusion-based morphogen shuttling that progresses through several nuclear

20 divisions. Perturbed shuttling in wntD mutant embryos results in a flat activation peak and

21 aberrant gastrulation. Re-entry of Dl into the nuclei at each cycle refines the signaling output,

22 by guiding graded accumulation of the T48 transcript that drives patterned gastrulation. We

23 conclude that diffusion-based ligand shuttling, coupled with dynamic readout, establishes a

24 refined pattern within the diffusible environment of early embryos. 

Introduction

27 The crude onset and subsequent refinement of spatial information shapes the future body 28 pattern of embryos. Morphogens, key instructive elements in this context, are secreted 29 signaling molecules that induce cells to adapt different fates depending on their concentration.

30 Establishing a morphogen gradient over a field of naïve cells patterns the cell layer into distinct 31 domains of gene expression (Green and Sharpe, 2015; Wolpert, 1971). Different strategies to 32 guide morphogen distribution have been identified. A common option is to produce the 33 morphogen in a restricted group of cells, giving rise to its graded distribution in the 34 surrounding cells (Lecuit et al., 1996; Nellen et al., 1996). Notably, in this scenario, the 35 morphogen-producing cells are distinct from the responding cells.

36 An alternative strategy of morphogen distribution is applicable to situations where the 37 morphogen is broadly expressed, and the gradient is generated within the field of expressing 38 cells, which also respond to the morphogen. This scenario is applicable to early embryos, 39 where broad transcriptional domains have been established, but have not yet given rise to the 40 determination of sufficiently restricted groups of cells, which could provide a local morphogen 41 source. In such situations, restricting morphogen signaling to a narrow domain becomes a 42 challenge, as diffusion tends to spread, rather than restrict ligand distribution.

43 Studies in several systems identified the Shuttling mechanism as a robust solution to this 44 challenge (Shilo et al., 2013). Here, a morphogen gradient is established not merely by its 45 diffusion away from the production source, but through an effective translocation of the 46 morphogen into the center of the field. This translocation, which is purely diffusion driven, is 47 mediated by a proximally-produced inhibitor. The resulting gradient is sharp and robust, 48 displaying limited sensitivity to gene dosages or reaction rate constants. Shuttling provides 49 robustness by concentrating the morphogen into restricted domain, which allows storing excess 50 levels in regions of maximal signaling without modifying the resulting cell fates. Such a 51 shuttling mechanism establishes the bone morphogenetic protein (BMP) morphogen gradient 52 in the early embryos of Drosophia and other insects (Eldar et al., 2002; Lapraz et al., 2009; 53 Shimmi et al., 2005; van der Zee et al., 2006; Wotton et al., 2017). It is also used for forming 
54 the BMP gradient in the Xenopus embryo, where it acquired additional features that allow 55 scaling of the gradient with embryo size (Ben-Zvi et al., 2014; Ben-Zvi et al., 2008).

56 Compelling evidence for shuttling was provided by comparing mutant phenotypes with the 57 predictions made by computational models (Ben-Zvi et al., 2008; Eldar et al., 2002; Haskel58 Ittah et al., 2012). It was also demonstrated that ligand produced ectopically in one part of the 59 embryo can be translocated to and endocytosed in the normal activation domain (Reversade 60 and De Robertis, 2005; Wang and Ferguson, 2005). Experimentally, these data were obtained 61 through the analysis of fixed embryos. Yet, the essence of the shuttling mechanism resides in 62 its dynamics. What is the time-frame during which the gradient is established? How fast is 63 gradient formation relative to its readout? Is the gradient stably formed, or is it subject to 64 subsequent cycles of refinements? Insight into these questions requires monitoring the dynamic 65 distribution of the morphogen within single embryos.

66 Furthermore, the shuttling mechanism makes a number of counter-intuitive predictions 67 regarding the dynamics of pattern formation. In particular, it predicts that signaling at the edge 68 of the source will initially increase, as ligand begins to accumulate, but will subsequently be 69 reduced, since ligand is continuously being shuttled to the center of the field. This non70 monotonic behavior is a defining property of the shuttling mechanism that concentrates ligand, 71 but is absent from other diffusion-based mechanisms establishing a graded pattern. In a certain 72 parameter range, shuttling also predicts transient formation of a double-peak pattern within the 73 gradient, again a prediction that is absent from naïve gradient-forming mechanisms. 74 Uncovering such features is again possible only by monitoring the dynamics of gradient 75 formation in live embryos.

76 The ability to observe the dynamics of morphogen gradient formation is challenging. The 77 ligands typically function at low levels. Furthermore, the morphogen may be present not only 78 in its active form, but also in a non-processed, inactive form, or bound to an inhibitor. Finally, 79 the morphogen is present in both extra- and intra-cellular locations. Most studies therefore 80 follow the patterning processes by quantifying the intracellular outcome of morphogen 81 signaling as a proxy for active morphogen distribution, using antibodies against the activated 82 (e.g, phosphorylated) states of signaling pathways triggered by morphogens (Dorfman and 
83 Shilo, 2001; Gabay et al., 1997; Tanimoto et al., 2000). Such approaches, however, cannot be 84 used to follow live embryos as they rely on immunostaining of fixed samples. An alternative is 85 to follow the transcriptional outcomes of morphogen signaling, but this analysis is already 86 quite removed from the original morphogen gradient itself, and is compounded by additional 87 regulatory inputs controlling the expression of the target genes.

88 Pattering the dorso-ventral (D-V) axis of the Drosophila embryo provides a powerful system to 89 analyze the dynamics of morphogen gradient formation. The early Drosophila embryo is a 90 syncytium, that is, a collection of nuclei that occupy a common cytoplasm enclosed by the 91 embryonic plasma membrane. Within this syncytium, the nuclei undergo 13 rapid and 92 synchronous divisions without any change in embryo size or shape. During the final four 93 division cycles, the nuclei form a monolayer just beneath the plasma membrane. Spaetzle (Spz) 94 is secreted from the syncytium into the surrounding peri-vitelline fluid, positioned between the 95 plasma and vitelline membranes, and a gradient of active Spz forms within it, along the radial 96 D-V axis. The processed Spz morphogen binds the transmembrane Toll receptor (DeLotto and 97 DeLotto, 1998; Morisato and Anderson, 1994; Schneider et al., 1994; Weber et al., 2003), and 98 triggers Dorsal (D1) translocation into the syncytial nuclei (Figure 1A). The processed form of 99 the Spz ligand therefore functions as the morphogen at this stage.

100 We previously showed that graded active Spz distribution is established by a shuttling 101 mechanism. In this case, shuttling is implemented in a self-organized manner through a 102 complex interplay between the active ligand and its pro-domain, which can accommodate 103 diverse structures (Haskel-Ittah et al., 2012; Shilo et al., 2013). The resulting gradient of active $104 \mathrm{Spz}$ is sharp, and provides robustness to a variety of perturbations in the level of pathway 105 components.

106 Entry of D1 into the nuclei can be followed in single live embryos carrying a Dl-GFP fused 107 protein (DeLotto et al., 2007). In this work, we use Light Sheet fluorescence microscopy for 108 live imaging of Dl-GFP nuclear localization during the final nuclear division cycles of the 109 syncytial Drosophila embryo. The resulting dynamics shows the two signatures of ligand 110 shuttling: a transient increase in signaling in the lateral regions, which is then reduced so as to 111 preferentially increase signaling at the ventral midline, and the resolution of two lateral peaks 
112 to a single central peak. We find that ligand shuttling is an ongoing process, which repeats

113 itself following each nuclear division. During the beginning of nuclear cycle (NC) 14, the

114 resulting dynamics of nuclear re-entry of Dl allows to further refine the resulting spatial

115 pattern, by triggering different temporal onsets of zygotic target gene expression in closely

116 positioned nuclei, thereby leading to a functionally significant graded accumulation of target

117 gene transcripts. In whtD mutant embryos, the Dl peak becomes flattened, and leads to an

118 abnormal increase in the number of cells simultaneously undergoing the initial step of

119 gastrulation, underscoring the significance of timely and properly shaped gradient formation.

120 Thus, diffusion-based ligand shuttling, coupled with a dynamic readout, establishes a

121 refined pattern within the environment of early embryos.

\section{Results}

\section{Temporal evolution of the Spz gradient during nuclear cycles 12-14}

125 Using Light Sheet fluorescence microscopy, we followed individual embryos carrying a Dl-

126 GFP fusion protein expressed under the endogenous $D l$ promoter (Figure 1B, SI: movie 1).

127 Consistent with previous reports (DeLotto et al., 2007; Kanodia et al., 2009), we observed a D-

$128 \mathrm{~V}$ gradient of nuclear Dl-GFP already at NC 12. This gradient was further refined and

129 elaborated during the next two cycles. To enable quantitative analysis of Dl-GPF nuclear

130 dynamics, we used an area preserving transformation to project the 3D images onto a 2D sheet.

131 We restricted our analysis to a region surrounding the A-P midline, where distortion due to 2D

132 projection is negligible (Figure 1C, SI: movie 2) (Heemskerk and Streichan, 2015). Next, we

133 automatically segmented the nuclei and averaged the nuclear Dl-GFP signal in nuclei

134 occupying a similar D-V axis position.

135 Our measurements defined the quantitative, spatio-temporal dynamics of D1-GFP at a 1-2 136 minute time resolution (Figure 1C-D, SI: movie 3, Methods). This dynamics results from the 137 extracellular active Spz gradient. However, inferring the profile of this extracellular gradient

138 from Dl-GFP dynamics is confounded by the fact that Dl-nuclear accumulation is established 139 anew at every nuclear cycle, since Dl exits the nucleus at mitosis upon nuclear envelope 
140 breakdown. We therefore needed a framework to suitably infer properties of the extracellular 141 active Spz gradient, and critically distinguish between models of gradient formation.

142 Toll signaling, at each given position along the D-V gradient, triggers the level of nuclear D1 143 and the rate by which this level increases. Thus, at the beginning of each division cycle, 144 following re-establishment of the nuclear envelope, nuclear D1 levels increase at a rate that is 145 proportional to the level of nearby Toll signaling. Conversely, at longer times, nuclear D1 146 levels approach a steady state, and are proportional to the extracellular Toll signaling. We 147 therefore plotted the dynamics of both parameters, nuclear Dl levels and its temporal change 148 during the onset of NC 14. Notably, we observe that this qualitative dynamics differs, 149 depending on the spatial position of nuclei along the D-V axis. In the ventral-most regions they 150 increased monotonically. In contrast, in lateral domains nuclear Dl displayed an overshoot, 151 initially increasing but then starting to decrease (Figure 2A-D, Figure S1). Clearly, such a 152 decrease in nuclear Dl is only possible if Toll signaling at this position decreases as well. 153 Therefore, the data indicates that the external Spz gradient continues to evolve through the 154 early part of NC 14, showing a distinct position-dependent, non-monotonic temporal signature.

155 To more rigorously infer dynamic properties of the external gradient from the measured pattern 156 of nuclear D1, we used computer simulations, modeling Dl-nuclear entry while assuming 157 different temporal patterns of Toll signaling (Figure 2E-L). Specifically, we searched for a 158 qualitative signature that distinguishes between three scenarios: (1) constant Toll signaling; (2) 159 Toll signaling that is changing (increasing) monotonically in time, as expected in naïve 160 gradient-forming models; and (3) a non-monotonic increase in Toll signaling, the signature 161 found in lateral regions of gradients formed by the shuttling mechanism. Our simulations have 162 shown that these scenarios are best distinguished by comparing the temporal changes in 163 nuclear $\mathrm{Dl}(\mathrm{d}(\mathrm{Dl}) / \mathrm{dt})$ with the levels of nuclear Dl. In the first two cases - constant or 164 monotonically increasing Toll activity - the relation between these two parameters is 165 invariably linear or concave (Figure 2E-L). In contrast, in the presence of non-monotonic 166 shuttling-based dynamics, a convex relation is obtained, with a pronounced negative temporal 167 derivative at the lateral regions, where nuclear D1 levels are low (Figure 2M-P). 
168 The measured data is not consistent with the dynamic defined by constant, or monotonically

169 increasing Toll signaling. Rather, it shows a clear signature of non-monotonic, shuttling-like

170 dynamics. Extending our simulations to include the full shuttling model that establishes the

171 active Spz gradient combined with Dl nuclear transport (See SI), confirmed that this model is

172 fully capable of simulating the experimentally observed dynamics, including the non-

173 monotonic, overshoot dynamics at the lateral regions.

174 An additional notable property of D1-nuclear entry dynamics was the initial formation, at every

175 nuclear cycle, of two ventro-lateral signaling peaks, that eventually converge to a single ventral

176 peak (Figures 1D, 2A, Figure S1). Thus, by 10-15 minutes into NC 14, when the major target

177 genes for Dl are induced, the initial two-peak gradient has refined to a single sharp peak. The

178 initial two-peak pattern provides another unique signature of shuttling-like dynamics. It is

179 expected under certain shuttling parameters, when the mean distance traveled by the shuttling

180 complex before it is cleaved, is much smaller than the distance to the ventral-most site. In this

181 case, ligand will initially accumulate at lateral regions, followed by gradual ventral

182 translocation (See SI). The reappearance of the double peak at every nuclear cycle likely

183 reflects a process of extracellular ligand mixing in the peri-vitelline fluid, possibly caused by

184 reorganization of the cortical actin-based cytoskeleton and deformation of the plasma 185 membrane associated with the nuclear divisions (di Pietro and Bellaiche, 2018; Zhang et al., 186 2018).

187 In conclusion, the dynamic behavior of Dl-GFP supports a continuous process of extracellular

188 Spz shuttling, displaying two of its defining signatures: non-monotonic dynamics of nuclear D1

189 entry in lateral regions, and the transient formation of two-peak gradient.

190 Altered shuttling dynamics in wntD mutants affects gastrulation

191 Dl-nuclear localization dynamics can be used for refined analysis of informative mutant 192 phenotypes. We applied this approach to study WntD, an inhibitor of Toll signaling which 193 provides a negative feedback that buffers the D-V patterning gradient against fluctuations 194 (Rahimi et al., 2016). wntD, a target of the Toll pathway, is transcribed locally at the posterior 
195 terminus of the embryo, and the secreted protein diffuses within the peri-vitelline fluid to 196 attenuate Toll signaling (Figure 3A)(Helman et al., 2012).

197 Genetic epistasis experiments have demonstrated that WntD binds the Frizzled-4 (Fz4) 198 receptor, and associates with the extracellular domain of Toll (Rahimi et al., 2016)(Figure 3B).

199 However, the consequences of this inhibition on gradient formation remained unclear. One 200 option is that WntD uniformly decreases signaling in all regions. Alternatively, with the 201 shuttling mechanism in mind, our simulations suggested that the binding of WntD to the 202 extracellular domain of Toll promotes ligand shuttling. This is because the binding of WntD to 203 the Toll receptor decreases the number of available receptors. This, in turn, compromises the 204 ability of free Spz to bind Toll, thereby increasing the probability that it will bind the free 205 shuttling molecule. Binding of Spz to Toll will thus be directed to more ventral regions, where 206 the levels of the shuttling molecules are sufficiently low. Uniform distribution of moderate 207 WntD levels would therefore lead to redistribution of the ligand to more ventral regions, 208 impacting not only on the strength of Toll signaling, but also on its sharpness (see Figure 3C,F 209 for simulation results).

210 Using live imaging in a wntD mutant background, we directly tested the possibility that WntD 211 promotes shuttling. Indeed, in wntD mutant embryos shuttling was less efficient. First, the 212 initial signature of a double peak was more prominent and persistent. Second, in contrast to $w t$ 213 embryos where gradual sharpening of the gradient takes place, in a wntD-mutant background 214 the final gradient was relatively wide and displayed a flattened single peak (Figures 3C-J, S2) 215 - consistent with the predicted role of WntD in redistribution of the Spz ligand (Figure 3K-L).

216 To follow the morphological consequences of a wider peak distribution of nuclear D1 217 distribution, we monitored wntD mutant embryos for an extended period of NC 14, observing 218 the processes of gastrulation and ventral furrow formation. We defined the edges of the 219 furrowing domain by marking the two lateral-most nuclei that alter their orientation upon 220 gastrulation. Working backwards to an earlier phase of NC 14, when the nuclei are still in a 221 monolayer, we can accurately count the number of nuclei between these edges. In contrast to 222 gastrulating $w t$ embryos where the initial invagination is observed in $\sim 9$ cells, in $w n t D$ mutants 223 a broader front of up to 15 cells invaginated at the same time (Figure 4). Thus, the shape of the 
224 Dl-activation gradient is essential for normal patterning and gastrulation. When the final 225 gradient peak is not sharp, a larger cohort of ventral cells takes part in furrow formation.

\section{Timing of wntD transcription}

227 wntD mutants display perturbed D1 dynamics already at NC 13 (Figures 3H, S3) suggesting

228 that WntD normally exerts its modulating effects at this early stage. This implies that zygotic 229 expression of $w n t D$, its translation, secretion to the peri-vitelline fluid and diffusion of the 230 protein, have commenced by then. To examine that this is possible, we applied our Light

231 Sheet-based visualization setup as a more sensitive assay for defining the onset and temporal 232 dynamics of whtD expression.

233 wntD expression is activated by nuclear D1, and is restricted to the posterior region, where 234 Torso signaling relieves Capicua (Cic) repression (Helman et al., 2012). We generated a 235 wntD::MS2 reporter, utilizing the genomic upstream regulatory sequence of wntD (Figure 5A). 236 The early expression profile of the reporter at the posterior part of the embryo mimics the 237 known pattern of wntD (Rahimi et al., 2016). Importantly, all eight embryos examined 238 expressed wntD, implying that overshooting of Toll signaling, which triggers expression of the 239 WntD "buffer", is a common consequence of D-V gradient signaling. When monitoring the 240 dynamics of $w n t D$ expression, the number of active nuclei transcribing wntD decreased 241 continuously between NCs 12 and 14 (Figure 5B-F). These results suggest that the WntD 242 protein already exerts its attenuating effect on Toll signaling at an early time.

243 Dl nuclear re-entry promotes graded expression of the zygotic $T 48$ gene by different 244 temporal onsets of transcription

245 The gradient of Dl-nuclear localization defines three major domains of zygotic gene expression 246 along the D-V axis. Within each of these domains several target genes are uniformly 247 expressed. The mesoderm is defined by highest levels of nuclear D1 and uniform expression of 248 the zygotic target genes twist (twi) and snail (sna) (Rusch and Levine, 1996). However, a 249 graded zygotic response within the mesoderm is also required: A gradient of apical myosin II 250 recruitment, peaking at the ventral midline, is essential for the ordered apical cell constriction 251 driving ventral furrow formation (Heer et al., 2017). A zygotic target gene that may lead to 
252 graded myosin II distribution is T48, since the T48 protein recruits Rho GEF2 to the apical 253 membrane, triggering the accumulation and contractile activity of an apical actomyosin 254 network (Kolsch et al., 2007). We asked whether the dynamics of Dl-nuclear entry may play a 255 role in generating a graded transcriptional response within this region, by following the zygotic 256 target gene T48.

257 Previous analysis of an MS2 reporter for T48 transcription demonstrated that the ventral-most 258 nuclei initiate transcription earlier than the lateral ones (Lim et al., 2017). Using the same 259 reporter, we find that signal intensity in transcribing nuclei is similar regardless of their 260 position along the $\mathrm{D}-\mathrm{V}$ axis, suggesting that once $T 48$ transcription is initiated, it progresses at 261 a constant rate in all nuclei (Figure 6C). If zygotic expression of Dl-target genes depends not 262 only on the final, steady-state level of nuclear Dl, but also on the dynamic profile of its 263 accumulation, the signaling output could be further sharpened. A dynamic phase of Dl nuclear 264 entry takes place during the initial 20 minutes of NC 14 (Figure 1D), the nuclear cycle 265 associated with a major onset of zygotic gene expression. A consequence of these dynamics is 266 that ventral-most nuclei will reach the threshold for expression of a given zygotic gene earlier 267 than more lateral ones. These ventral nuclei will begin to express the gene earlier, therefore 268 expressing it for a longer period than more lateral nuclei, and could thus accumulate more 269 transcripts.

270 To examine the consequences of the graded onset of T48 transcription on mRNA 271 accumulation, we carried out quantitative single-molecule FISH using T48 probes. The signal 272 obtained is comprised of two components: Prominent puncta in the nuclei representing active 273 transcription of the gene, and sparse weaker spots in the cytoplasm marking accumulated 274 individual mRNA molecules. We analyzed embryos that demonstrated ongoing transcription in 275 several nuclear rows along the D-V axis. The number of transcribing nuclei in ventral and 276 lateral positions within the expression domain was similar, and the transcription intensity in the 277 different rows appears comparable. This again indicates that once $T 48$ transcription is initiated, 278 it progresses at a constant rate in all nuclei, regardless of position (Figure 6D-E). We next 279 quantitated the levels of cytoplasmic T48 mRNA, using the TransQuant (Bahar Halpern and 280 Itzkovitz, 2016). A clear D-V gradient of cytoplasmic mRNA accumulation is observed across 281 several cell rows, peaking at the ventral midline (Figure 6F-G). This result indicates that the 

T48 mRNA is sufficiently stable during the temporal window of early NC 14, such that the 283 time of onset of its transcription along the D-V axis, governed by the dynamics of Dl nuclear 284 entry, correlates with the level of mRNA that accumulates in the adjacent cytoplasm. D1 285 nuclear entry dynamics thus appear to be a critical factor regulating graded T48 activity along 286 the D-V axis.

\section{Discussion}

\section{Dynamics of the Spz extracellular morphogen gradient}

289 The early Drosophila embryo provides extreme challenges for the generation and maintenance

290 of extracellular morphogen gradients. Most notably, the peri-vitelline fluid surrounding the 291 embryo facilitates rapid diffusion of molecules (Stein et al., 1991). In addition, the alteration in 292 the surface of the plasma membrane at every nuclear division provides an active mixing force 293 (di Pietro and Bellaiche, 2018; Zhang et al., 2018). Thus, analysis of the early morphogen 294 gradients operating in this environment, including ventral Spz/Toll activation and the 295 subsequent BMP gradient patterning the dorsal aspect, should consider this highly dynamic 296 environment. In the case of the Toll pathway, the active Spz ligand is generated by proteolytic 297 processing within the extra-embryonic peri-vitelline fluid in a broad ventral region, defined by 298 the activation domain of the Easter (Ea) protease (Cho et al., 2012). The generation of a sharp 299 Spz activation gradient within this broad ventral domain of processing takes place by 300 diffusion-based shuttling. Our previous work demonstrated that the pro-domain of Spz plays an 301 instructive role in delivering the active, cleaved ligand towards the ventral midline (Haskel302 Ittah et al., 2012). While a variety of experiments and computational analyses indicated the 303 utilization of a "self-organized shuttling" mechanism in this context, it was imperative to 304 visualize the actual dynamics of the process.

305 We were able to infer the dynamics of the extracellular Spz gradient by following the kinetics 306 of Dl-GFP nuclear accumulation in individual live embryos during the final syncytial nuclear 307 division cycles and the early phase of NC 14. Nuclear levels of Dl are not a direct readout of 308 the extracellular gradient, since accumulation of D1 in the nuclei is re-initiated at the onset of 309 every nuclear cycle. Nevertheless, it is possible to infer key features of the extracellular Spz 
310 gradient from this dynamic behavior. Using this approach we identified clear hallmarks of

311 ligand shuttling, most notably the lateral overshoot and the presence of two lateral peaks which

312 converge to a central ventral peak. This convergence takes place within a timeframe of

313 minutes, and repeats at every nuclear cycle. Since new protein molecules of the extracellular

314 components are continually translated, the ongoing activity of the shuttling process is vital.

315 Therefore, shuttling is important not only for generating the gradient, but also for maintaining

316 it, in the face of rapid diffusion and mixing within the peri-vitelline fluid. Importantly, by $\sim 10$ -

31715 minutes into NC 14, when the robust induction of transcription of the cardinal zygotic Dl-

318 target genes twt and sna ensues, the nuclear gradient of Dl is sharp and a single ventral peak is

319 resolved.

320 The role of WntD in shaping and buffering the Spz gradient

321 Having described the dynamics of Dl-nuclear entry and gradient formation, we were in a 322 position to use our experimental approach in order to examine regulatory processes affecting 323 Toll signaling. The Wnt family ligand WntD provides an essential buffering system to 324 variations in Toll signaling between embryos (Rahimi et al., 2016). wntD is an early zygotic 325 gene that is expressed initially at the posterior-ventral region of the embryo, and its expression 326 levels depend on the magnitude of Toll signaling (Helman et al., 2012). Although WntD is 327 produced locally, the rapid secretion and diffusion of the protein in the peri-vitelline space 328 generates a uniform attenuation of Toll signaling throughout the embryo surface. The activity 329 of WntD leads in different embryos to convergence of the variable global Toll activation 330 gradient to a similar pattern, which is dictated by the fixed final signaling level that shuts off 331 wntD expression (Rahimi et al., 2016). We term this paradigm “distal pinning”, achieved in 332 this case by an induction-contraction mechanism (Shilo and Barkai, 2017).

333 Secreted WntD is recruited to the plasma membrane by binding to its receptor Fz4. Epistasis 334 assays have indicated that WntD exerts its inhibitory effect on Toll signaling by associating 335 with the extracellular domain of Toll (Rahimi et al., 2016), thereby reducing the number of 336 Toll receptors that are available for binding Spz. Bearing the cardinal features of shuttling in 337 mind, this mode of inhibition implies that the effect of WntD would be global and non- 
338 autonomous, and will actually change the shape of the gradient, making it sharper. The

339 observed dynamics of Dl-GPF in wntD mutant embryos indeed confirms this prediction.

340 The shuttling process is driven by competition between the inhibitory Spz pro-domain and the

341 Toll receptor for binding free, active Spz. Binding to the pro-domain is favored in the lateral

342 part of the embryo, where its concentration is higher, while in more ventral regions binding to

343 Toll takes over. Since WntD impinges on the extracellular properties of the Toll receptor, the

344 active ligand is deposited in more ventral regions, where the concentration of the pro-domain is

345 lower. Thus, WntD does not simply reduce the overall profile of Toll activation, but actually

346 re-directs the ligand from the lateral regions to the ventral domain. We have previously shown

347 that accumulation of excess ligand in the peak by shuttling is an effective mechanism to buffer

348 noise. Since activation in this region is already maximal, the excess ligand will not alter the

349 resulting cell fates (Barkai and Shilo, 2009).

350 The rapid timing of processes in the early embryo and the short duration of interphases

351 between nuclear divisions raises the question of whether it is actually possible to produce

352 sufficient levels of WntD that will drive the morphogen profile to the desired equilibrium.

353 When monitoring wntD transcription directly utilizing the MS2 system, we saw that most, if

354 not all embryos express wntD, indicating that Toll signaling overshoots in most embryos.

355 Furthermore, within single embryos the number of nuclei expressing wntD was reduced

356 between NCs 12 and 13, and completely terminated by NC 14, implying that WntD impinges

357 on the Toll gradient and its own expression by this time. The intronless arrangement of the

358 wntD gene and the rapid secretion of the protein, which does not require post-translational

359 modifications (Herr et al., 2012), may facilitate the process.

\section{DI nuclear re-entry refines its signaling output}

361 The ventral cohort of zygotic target genes including twi and sna is induced by the Toll 362 activation gradient, and the threshold for their induction corresponds to $\sim 50 \%$ of maximal Dl363 nuclear localization (Kanodia et al., 2009; Liberman et al., 2009). Within the ventral domain, 364 nuclei exhibit a similar level of sna transcription (Bothma et al., 2015; Lagha et al., 2013). 
365 These genes are triggered at NC 14 after the Dl gradient is stabilized and a distinct activation 366 peak generated.

367 Are there zygotic target genes that respond to the dynamics of Dl nuclear targeting, before it 368 stabilizes? This appears to be the case for $T 48$, which encodes a transmembrane protein that 369 facilitates recruitment of RhoGEF2 and ultimately Rho and actomyosin, to mediate apical 370 constriction of invaginating mesodermal cells (Kolsch et al., 2007). Graded distribution of 371 myosin II was shown to be critical for proper invagination of these cells to form the ventral 372 furrow (Heer et al., 2017).

373 We provide evidence that the graded distribution of T48 mRNA results from the dynamics of 374 Dl-nuclear re-entry at NC 14. The ventral-most cells reach the threshold of T48 induction 375 earlier than more lateral cells, and hence will express the gene longer (Lim et al., 2017). 376 Integration of the length of expression along the $\mathrm{D}-\mathrm{V}$ axis then leads to a gradient of 377 cytoplasmic T48 mRNA accumulation. This example represents a unique case, where graded 378 morphogen activation instructs the generation of a gradient of target-gene expression. The 379 strict dependence on the timing of transcription initiation provides another mechanism to 380 generate differences between adjacent nuclei along the D-V axis.

381 In conclusion, this work has utilized live imaging of Toll pathway activation, to identify and 382 characterize the hallmarks of ligand shuttling (Figure 7). This process is rapid and takes place 383 continuously throughout the final nuclear division cycles, to generate and maintain a sharp 384 activation gradient in the diffusible environment of the peri-vitelline fluid. WntD impinges on 385 Spz shuttling, and is responsible not only for buffering variability between embryos, but also 386 for generating a sharp activation peak. This peak is utilized to induce a graded expression of a 387 zygotic target gene that is essential for executing processes that drive gastrulation. Thus, 388 diffusion-based ligand shuttling, coupled with a dynamic readout, establishes a refined pattern 389 within the environment of early embryos. 


\section{Acknowledgements}

394 We thank B. Lim and M. Levine for the T48::MS2 flies, and S. Itskovitz for advice on single-

395 molecule FISH. We are grateful to Y. Addadi and O. Golani for help in acquisition and 396 analysis of Light Sheet images, and S. Streichan for support in 3D image processing. Imaging 397 using the Light Sheet microscope was made possible thanks to The de Picciotto-Lesser Cell 398 Observatory in memory of Wolf and Ruth Lesser. We thank the members of the Shilo and 399 Barkai labs for fruitful discussions. The work was supported by an ERC advanced grant to 400 N.B. and a US-Israel Binational grant to E.S. and B.S. B.S. is an incumbent of the Hilda and 401 Cecil Lewis chair in Molecular Genetics.

402

403 Methods

\section{$404 \quad$ Fly stocks and genetics}

405 For the $w t$ dlGFP experiments we used Sco/Cyo;dl-GFP/Tm3, Sb flies (DeLotto et al., 2007). 406 For the wntD mutant background, a recombination between a wntD null allele (Rahimi et al., 407 2016)and $d l-G F P$ was carried out, and crossed to whtD mutant.

408 wntD::MS2-Fhos-RH generation: the 1162 bp upstream of wntD transcription start site were 409 synthesized followed by 24 repeats of the MS2 sequence, and placed within the 5UTR. The 410 sequence was inserted into a pAttB vector with NotI and KpnI sites. The Fhos-RH sequence 411 was further ligated into the NotI site to generate wntD::MS2-Fhos-RH. Virgin females 412 expressing both MCP::GFP and His:RFP were crossed with males of the reporter line 413 whtD::MS2-Fhos-RH to collect embryos for imaging.

414 MCP::GFP and t4::MS2-yellow fly lines (Lim et al., 2017) used in the quantitative live imaging 415 of T48 induction were kindly provided by B. Lim. Virgin females carrying both MCP::GFP 416 and His:RFP were crossed with males of the reporter line T48::MS2-yellow and embryos 417 collected for imaging. 


\section{Live Imaging}

420 Embryos were imaged using a Light Sheet z1 microscope (Zeiss Ltd.) equipped with 2 sCMOS

421 cameras PCO-Edge, 10X excitation objectives and Light Sheet Z.1 detection optics 20×/1.0

422 (water immersion). The embryos were collected, dechorionated and up to 4 embryos were 423 sequentially mounted perpendicularly into a glass capillary (Brand) in a 1\% low melting 424 agarose solution (Roth). Imaging was preformed using dual side illumination, zoom X0.8. GFP 425 Excitation: 488nm Emission / detection - BP 505-545, RFP Excitation: 561nm Emission / 426 detection - BP 575-615.

427 The T48::MS2 expressing embryos were mounted on a cover slide and imaged through 428 halocarbon oil in a Zeiss LSM710 confocal system at a temporal resolution of 3 minutes.

\section{MS2 analysis}

430 MCP-GFP spots were manually counted using the Imaris software after adjusting the contrast 431 min\&max for enhanced visualization. In the T48::MS2 analysis the spots were manually 432 detected and displayed using the Imaris spots object.

\section{3 sm-FISH}

434 Stellaris RNA FISH probe sets for the T48 gene (5' UTR and coding, not including the 3' 435 UTR), were designed by Stellaris Probe Designer and purchased from LGC Biosearch 436 Technologies. $3 \mathrm{hrs}$ after egg lay (AEL) $w t$ embryos were fixed for $25 \mathrm{~min}$ in $4 \%$ 437 formaldehyde, washed in Methanol and kept at $-20^{\circ} \mathrm{C}$. Next day embryos were washed in 438 Methanol and then in Ethanol, rocked in 90\% Xylene, 10\% Ethanol for $1 \mathrm{hr}$ followed by post 439 fixation. Then incubated 6' with Proteinase K and post fixed again. Embryos were transferred 440 gradually to $10 \% \mathrm{FA}$ in $2 \mathrm{X} \mathrm{SSC}+10 \mu \mathrm{g} / \mathrm{ml}$ ssDNA preheated to $37^{\circ} \mathrm{C}$ and prehybredized for $44130^{\prime}$ at $37^{\circ} \mathrm{C}$. Hybridization Buffer included 10\% FA, 10\% Dextran, 2mg/ml BSA, RVC and 442 ssDNA+ tRNA in $2 \mathrm{X}$ SSC, containing the probe set (1 ng/ $\mu \mathrm{l})$ (Trcek et al., 2017). 443 Hybridization was carried out $\mathrm{O} / \mathrm{N}$ at $37^{\circ} \mathrm{C}$. Next morning the embryos were shaken gently and 444 incubated for another $30^{\prime}$. Embryos were washed twice for $30^{\prime}$ at $37^{\circ} \mathrm{C}$ with $10 \% \mathrm{FA}$ in $2 \mathrm{X}$ $445 \mathrm{SSC}+10 \mu \mathrm{g} / \mathrm{ml}$ ssDNA and gradually transferred to PBS-0.5\% Tween and mounted with 446 Vectashield+DAPI Mounting Medium (Vector Laboratories Inc.). Fluorescence was visualized 
447 with a Nikon Eclipse Ti2 microscope, and analyzed by the TransQuant script as was previously 448 published (Bahar Halpern and Itzkovitz, 2016). TS Intensities were measured via ImageJ.

\section{$449 \quad$ Light Sheet movies analysis}

\section{Projection to 2D}

451 To enable quantitative analysis of the nuclear Dl gradient, we projected the 3D scans of the 452 embryo from the light-sheet microscope, into a 2D flat surface, for every time point imaged.

453 This was possible, since all the nuclei are arranged on the surface of the embryo, whose shape 454 resembles an ellipsoid. This ellipsoid can be projected into a 2D surface, which contains all the 455 nuclei and therefore the entire nuclear D1 gradient. To this end, we used an area preserving 456 transformation with minimal distortion far from the Anterior and Posterior poles, implemented 457 by the IMSANE tissue cartography tool (Heemskerk and Streichan, 2015). IMSANE was used 458 with the following specifications: Planar Illastik surface detector and cylinder chart type. 459 Surface detection was performed on the last time point for each embryo, and the detected 460 surface was then used to project all earlier time points. Since the embryo is, to a good 461 approximation, a cylinder apart from the anterior and posterior poles, embryo circumference 462 was defined as the largest circumference of the ellipsoid fitted to the embryo surface by 463 IMSANE.

\section{Nuclei segmentation}

465 The nuclei were detected separately for each time point, using the following segmentation 466 method:

1. Automated local thresholding of the image in order to create a binary mask. Done in Image J using the Bernsen algorithm with a contrast threshold of 15.

2. The resulting binary mask underwent further refinement to segment the nuclei using MATLAB image analysis filters:

a. All connected objects in the mask large enough to be nuclei (over 50 pixels in size) were located and classified into 3 size groups: small 50-150 pixels, medium 150-600 pixels and large 600 pixels and over.

b. Each size group underwent erosion using imerode and then dilation using imdilate with appropriate filter sizes for each group. 

and dilation with same filter size as first round.

3. The resulting objects in the binary mask were filtered by size to exclude objects too small (under 50 pixels) or too large (over 3000 pixels) to be a single nucleus.

\section{Measuring the nuclear DI gradient around the A-P midline}

482 Gradient measurement was performed by first manually discarding all time points between the 483 nuclear cycles. Then, for every detected nucleus, for all remaining time points, the value of 484 nuclear Dl was calculated as the mean intensity inside the nucleus (located by the above 485 segmentation method). The location of the A-P midline was manually selected for each 486 embryo. A rectangular area around the A-P midline was then defined. The width of the area 487 along the A-P axis was $15 \%$ of entire A-P length and it spanned the entire D-V axis. For NC14, 488 this definition corresponds to $\sim 8-10$ columns of nuclei closest to the A-P midline. Only the 489 nuclei inside this area were taken into account for gradient measurement, monitoring several 490 columns of nuclei along the A-P axis gave rise to averaging of D1-nuclear intensity along this 491 small window. The spatial axis for the gradient was defined as a relative axis- $x / L$, indicating 492 location on the $\mathrm{D}-\mathrm{V}$ axis- $x$ divided by embryo circumference- $L$. In order to assign a location 493 on this relative $\mathrm{D}-\mathrm{V}$ axis for each nucleus, the location of the $\mathrm{D}-\mathrm{V}$ midline was manually 494 selected and defined as $x / L=0$. This resulted in the raw intensity function, $D l_{\text {raw }}(x / L, t)$ 495 measuring nuclei intensity along the relative $\mathrm{D}-\mathrm{V}$ axis, over time. This function, $D l_{\text {raw }}(x / L, t)$,

496 was then smoothened in space, for each time point separately using the MATLAB smooth 497 function with a smoothing coefficient of 0.23 . The smoothened data was then fitted by a 498 smoothing spline using the MATLAB fit function and evaluated on a 1000 linearly spaced $499 x / L$ locations. The resulting function, $D l_{\text {smooth }}(x / L, t)$, was plotted in main text figures.

\section{Dorsal gradient as a function of time, at specific locations along the D-V axis}

501 For the calculation of Dl-nuclear intensity over time at a specific location $\tilde{x} / L$, we used $502 D l_{\text {smooth }}(x / L, t)$ at that location: $D l_{\text {smooth }}(\tilde{x} / L, t)$. Background subtracted values were 503 calculated separately for each NC, by subtracting the minimal intensity observed in a nucleus 504 for that NC. $D l_{\text {smooth }}(\tilde{x} / L, t)$ was then smoothened in time using the MATLAB smooth 
505 function with the loess method and a smoothing coefficient of 0.5. It was then fitted with a

506 smoothing spline, resulting in the function $D l_{\tilde{x} / L}^{\text {fitted }}(t)$. The temporal derivative of nuclear

507 Dorsal, at a specific location- $\tilde{x} / L$ was calculated by applying a third order finite differences

508 formula to $D l_{\tilde{x} / L}^{\text {fitted }}(t)$ and then smoothing using smooth with a smoothing coefficient of 0.6

509 and fitting a smoothing spline.

\section{$510 \quad$ Measuring peak sharpness}

511 The peak sharpness measure for an embryo was calculated based on the values of $D l_{\text {smooth }}(x /$

$512 L, t)$, around the ventral-most location. For each time point, $\tilde{t}, D l_{\text {smooth }}(x / L, \tilde{t})$ was

513 normalized by dividing by its maximal value at $\tilde{t}$. Peak sharpness was then calculated as the

514 standard deviation divided by the mean, in percent, of values close to the peak: within the

515 range $\frac{x}{L} \in[-0.1,0.1]$. This measure captures how different from each other are values close to 516 the peak. 
530 Bahar Halpern, K., Itzkovitz, S., 2016. Single molecule approaches for quantifying transcription and degradation rates in intact mammalian tissues. Methods 98, 134-142.

532 Barkai, N., Shilo, B.Z., 2009. Robust generation and decoding of morphogen gradients. Cold 533 Spring Harb Perspect Biol 1, a001990.

534 Ben-Zvi, D., Fainsod, A., Shilo, B.Z., Barkai, N., 2014. Scaling of dorsal-ventral patterning in the Xenopus laevis embryo. Bioessays 36, 151-156.

536 Ben-Zvi, D., Shilo, B.Z., Fainsod, A., Barkai, N., 2008. Scaling of the BMP activation gradient 537 in Xenopus embryos. Nature 453, 1205-1211.

538 Bothma, J.P., Garcia, H.G., Ng, S., Perry, M.W., Gregor, T., Levine, M., 2015. Enhancer additivity and non-additivity are determined by enhancer strength in the Drosophila embryo. protease in the Drosophila egg controls embryo dorsoventral polarity. Curr Biol 22, 10131018. shuttling mediates the dynamic maintenance of nuclear Dorsal levels during Drosophila embryogenesis. Development 134, 4233-4241. easter generates a dimeric NGF-like molecule with ventralising activity. Mech Dev 72, 141148.

552 Dorfman, R., Shilo, B.Z., 2001. Biphasic activation of the BMP pathway patterns the Dev Cell 45, 539-541.

561 Self-organized shuttling: generating sharp dorsoventral polarity in the early Drosophila Drosophila embryonic dorsal region. Development 128, 965-972.

Eldar, A., Dorfman, R., Weiss, D., Ashe, H., Shilo, B.Z., Barkai, N., 2002. Robustness of the BMP morphogen gradient in Drosophila embryonic patterning. Nature 419, 304-308.

Gabay, L., Seger, R., Shilo, B.Z., 1997. MAP kinase in situ activation atlas during Drosophila embryogenesis. Development 124, 3535-3541.

Green, J.B., Sharpe, J., 2015. Positional information and reaction-diffusion: two big ideas in developmental biology combine. Development 142, 1203-1211.

Haskel-Ittah, M., Ben-Zvi, D., Branski-Arieli, M., Schejter, E.D., Shilo, B.Z., Barkai, N., 2012. embryo. Cell 150, 1016-1028.

Heemskerk, I., Streichan, S.J., 2015. Tissue cartography: compressing bio-image data by dimensional reduction. Nat Methods 12, 1139-1142.

Heer, N.C., Miller, P.W., Chanet, S., Stoop, N., Dunkel, J., Martin, A.C., 2017. Actomyosinbased tissue folding requires a multicellular myosin gradient. Development 144, 1876-1886.

568 S.Y Paroush, Z, 2012. RTK signaling modulates the Dorsal gradient Development 139, 569 3032-3039.

570 Herr, P., Hausmann, G., Basler, K., 2012. WNT secretion and signalling in human disease.

571 Trends Mol Med 18, 483-493. 
572 Kanodia, J.S., Rikhy, R., Kim, Y., Lund, V.K., DeLotto, R., Lippincott-Schwartz, J., 573 Shvartsman, S.Y., 2009. Dynamics of the Dorsal morphogen gradient. Proc Natl Acad Sci U S 574 A 106, 21707-21712.

575 Kolsch, V., Seher, T., Fernandez-Ballester, G.J., Serrano, L., Leptin, M., 2007. Control of 576 Drosophila gastrulation by apical localization of adherens junctions and RhoGEF2. Science $577 \quad 315,384-386$.

578 Lagha, M., Bothma, J.P., Esposito, E., Ng, S., Stefanik, L., Tsui, C., Johnston, J., Chen, K., 579 Gilmour, D.S., Zeitlinger, J., Levine, M.S., 2013. Paused Pol II coordinates tissue 580 morphogenesis in the Drosophila embryo. Cell 153, 976-987.

581 Lapraz, F., Besnardeau, L., Lepage, T., 2009. Patterning of the dorsal-ventral axis in 582 echinoderms: insights into the evolution of the BMP-chordin signaling network. PLoS Biol 7, 583 e1000248.

584 Lecuit, T., Brook, W.J., Ng, M., Calleja, M., Sun, H., Cohen, S.M., 1996. Two distinct 585 mechanisms for long-range patterning by Decapentaplegic in the Drosophila wing. Nature 381, 586 387-393.

587 Liberman, L.M., Reeves, G.T., Stathopoulos, A., 2009. Quantitative imaging of the Dorsal 588 nuclear gradient reveals limitations to threshold-dependent patterning in Drosophila. Proc Natl 589 Acad Sci U S A 106, 22317-22322.

590 Lim, B., Levine, M., Yamazaki, Y., 2017. Transcriptional Pre-patterning of Drosophila 591 Gastrulation. Curr Biol 27, 610.

592 Morisato, D., Anderson, K.V., 1994. The spatzle gene encodes a component of the 593 extracellular signaling pathway establishing the dorsal-ventral pattern of the Drosophila embryo. Cell 76, 677-688.

Nellen, D., Burke, R., Struhl, G., Basler, K., 1996. Direct and long-range action of a DPP morphogen gradient. Cell 85, 357-368.

600 Reversade, B., De Robertis, E.M., 2005. Regulation of ADMP and BMP2/4/7 at opposite 2016. A WntD-Dependent Integral Feedback Loop Attenuates Variability in Drosophila Toll Signaling. Dev Cell 36, 401-414.

608 Shilo, B.Z., Barkai, N., 2017. Buffering Global Variability of Morphogen Gradients. Dev Cell 609 40, 429-438.

610 Shilo, B.Z., Haskel-Ittah, M., Ben-Zvi, D., Schejter, E.D., Barkai, N., 2013. Creating gradients 611 by morphogen shuttling. Trends Genet 29, 339-347.

612 Shimmi, O., Umulis, D., Othmer, H., O'Connor, M.B., 2005. Facilitated transport of a $613 \mathrm{Dpp} / \mathrm{Scw}$ heterodimer by Sog/Tsg leads to robust patterning of the Drosophila blastoderm 614 embryo. Cell 120, 873-886.

615 Stein, D., Roth, S., Vogelsang, E., Nusslein-Volhard, C., 1991. The polarity of the dorsoventral 616 axis in the Drosophila embryo is defined by an extracellular signal. Cell 65, 725-735. 
617 Tanimoto, H., Itoh, S., ten Dijke, P., Tabata, T., 2000. Hedgehog creates a gradient of DPP 618 activity in Drosophila wing imaginal discs. Mol Cell 5, 59-71.

619 Trcek, T., Lionnet, T., Shroff, H., Lehmann, R., 2017. mRNA quantification using single620 molecule FISH in Drosophila embryos. Nat Protoc 12, 1326-1348.

621 van der Zee, M., Stockhammer, O., von Levetzow, C., Nunes da Fonseca, R., Roth, S., 2006. $622 \mathrm{Sog} / \mathrm{Chordin}$ is required for ventral-to-dorsal Dpp/BMP transport and head formation in a short 623 germ insect. Proc Natl Acad Sci U S A 103, 16307-16312.

624 Wang, Y.C., Ferguson, E.L., 2005. Spatial bistability of Dpp-receptor interactions during 625 Drosophila dorsal-ventral patterning. Nature 434, 229-234.

626 Weber, A.N., Tauszig-Delamasure, S., Hoffmann, J.A., Lelievre, E., Gascan, H., Ray, K.P., 627 Morse, M.A., Imler, J.L., Gay, N.J., 2003. Binding of the Drosophila cytokine Spatzle to Toll 628 is direct and establishes signaling. Nat Immunol 4, 794-800.

629 Wolpert, L., 1971. Positional information and pattern formation. Curr Top Dev Biol 6, 183 630224.

631 Wotton, K.R., Alcaine-Colet, A., Jaeger, J., Jimenez-Guri, E., 2017. Non-canonical 632 dorsoventral patterning in the moth midge Clogmia albipunctata. Evodevo 8, 20.

633 Zhang, Y., Yu, J.C., Jiang, T., Fernandez-Gonzalez, R., Harris, T.J.C., 2018. Collision of 634 Expanding Actin Caps with Actomyosin Borders for Cortical Bending and Mitotic Rounding 635 in a Syncytium. Dev Cell 45, 551-564 e554. 


\section{Figure Legends}

639 Figure 1. Dynamics of Dl-nuclear localization. (A) Schematic cross-section of an early 640 embryo: Spz is distributed in a sharp gradient along the D-V axis within the peri-vitaline 641 space. Spz binding to the Toll receptor triggers a signaling cascade, culminating in a gradient 642 of nuclear D1 with a sharp ventral peak. Generation of the Spz sharp gradient was proposed to 643 employ a self-organized shuttling mechanism (Haskel-Ittah et al., 2012). (B) A frame from a 644 Light Sheet time-lapse movie following the dynamics of endogenously expressed Dl-GFP in 645 the entire embryo. The gradient can be visualized already at NC 12, it is lost during nuclear 646 divisions and is re-generated at the onset of each nuclear cycle. (C) Each frame of the 3D Light 647 Sheet scan was projected to a 2D map using the ImSAnE tool (Heemskerk and Streichan, 648 2015). Here, the $2 \mathrm{D}$ projection of the frame from $\mathrm{B}$ is shown. The quantification of Dl-GFP 649 intensity in the nuclei along the D-V axis at 1 min intervals was carried out on nuclei inside the 650 dashed frame. This region, which is close to the middle of the A-P axis, is not distorted by the 651 2D projection (See Methods). (D) Dl-GFP intensity, plotted as function of relative location 652 along the $\mathrm{D}-\mathrm{V}$ axis (relative location axis $\mathrm{x} / \mathrm{L}$ is defined as location divided by embryo 653 circumference (See Methods)). A curve is shown for each time point for NC 12, 13 and 14 for 654 the same $w t$ embryo. Each Dl-GFP intensity curve was smoothened and normalized by the 655 maximal value attained during NC14 (See Methods). Time points are one minute apart, going 656 from earliest time points in blue to the latest in red. Duration of each NC in minutes is 657 indicated above the plot.

658 Figure 2. Measured nuclear Dl dynamics during NC14 indicates ongoing shuttling. (A-D)

659 Measured nuclear Dl temporal dynamics for a $w t$ embryo, time points are color coded and are 660 one minute apart. (E-P) Simulations of nuclear Dl temporal dynamics (See SI). (E-H) 661 Diffusion model: nuclear D1 localization induced by Spz binding the Toll receptor, with Spz 662 diffusing out of a wide source while being degraded. (I-L) Constant external gradient model: 663 nuclear Dl localization induced by Spz binding the Toll receptor, with a Spz gradient that is 664 constant in time. (M-P) Full model: nuclear Dl localization induced by Spz binding the Toll 665 receptor, with shuttling of Spz. (A,E,I,M) Nuclear Dl levels in [a.u], plotted as function of 666 relative location along the $\mathrm{D}-\mathrm{V}$ axis. A curve is shown for each time point, time points are 
667 color coded. (B,F,J,N) Nuclear Dl levels at specific locations along the D-V axis, as function of 668 time. Nuclear localizations along the D-V axis are color coded. Each location's curve was 669 normalized by its own maximal value. (C,G,K,O) Nuclear D1 temporal derivative at specific 670 locations along the D-V axis, as function of time. Each location's curve was normalized by its 671 own maximal value. (D,H,L,P) Nuclear Dl temporal derivative at specific locations along the 672 D-V axis, as function of nuclear D1. Each location's curve was normalized by its own maximal 673 value. Color codes for the location of nuclei along the D-V axis and for the temporal dynamics 674 are shown.

675 Figure 3. Shuttling dynamics are impaired in the absence of WntD. (A) A scheme showing 676 the integral feed-back loop between Dl and WntD. wntD expression is restricted to the 677 posterior side of the embryo (purple) and is induced by D1. (B) A scheme showing WntD 678 binding to Fz4, and restricting the binding of Spz to the Toll receptor. (C,F) Simulations of D1 679 temporal dynamics in the full model for a $w t(\mathrm{C})$ and $w n t D$ mutant (F) embryo (See SI). Time 680 points are color coded. For the wntD mutant, the double peak is more prominent and does not 681 fully converge resulting in a flat peak. (D,G) Measured D1 temporal dynamics for a $w t$ (D) and 682 wntD mutant $(\mathrm{G})$ embryo (See Methods). Time points are color coded, and are one minute 683 apart. As predicted by the model, the wntD mutant exhibits less efficient shuttling leading to a 684 more prominent double peak, which does not fully converge, resulting in a flat peak. (E,H) Log 685 of Dl-GFP intensity temporal dynamics at selected locations along the D-V axis for a $w t$ (E) 686 and wntD mutant $(\mathrm{H})$ embryo from panels D,G. Locations are color coded. Measured values 687 were background subtracted and smoothed in time (See Methods). The flat peak of the wntD 688 mutant embryo results in very similar Dl values over time for the four ventral most curves, 689 while in the $w t$ embryo each curve attains a different final value and has different dynamics. 690 (I-J) Peak sharpness over time (calculated as std/mean in \% of values close to the peak, See 691 Methods) in a population of wt embryos (black) and wntD mutant embryos (red) during NC 13 692 (I) and NC14 (J). Bold lines indicate population mean and surrounding color indicates standard 693 error. Number of embryos in each population is indicted in plot. Sharpness in the $w t$ population 694 increases over time to a value significantly higher than the wntD mutant population. The wntD 695 mutant population exhibits an initial increase in peak sharpness due to the prominent double 696 peak, followed by a decrease due to peak flattening. (K) Scheme showing the global 
697 attenuation effect of WntD on Toll availability, leading to redirection of active ligand binding

698 towards more ventral positions. (L) In wntD mutants additional Toll receptors become

699 available, leading to increased ligand binding in lateral regions, generating a flatter peak of

700 signaling.

701 Figure 4. An enlarged ventral furrow is formed in the absence of WntD. Snapshots of 702 cross-sections of a $w t$ (A-D) vs. wntD mutant embryo (E-F) expressing Dl-GFP, showing the 703 ventral nuclei during NC 14. Time in NC 14 (in minutes) is indicated. When the first sign of 704 invagination appeared, the most lateral nuclei still displaying positional change were marked 705 (yellow). Working backwards in the movies allowed an accurate count of the nuclei between 706 them prior to invagination.

707 Figure 5. Dynamics of wntD transcription. (A) Schematic representation of the wntD::MS2 708 reporter. The $1.2 \mathrm{~kb}$ regulatory region upstream of the wntD coding sequence drives the 709 expression of Fhos-RH with 24 MS2 repeats located at its 5' end. (B) Light Sheet live imaging 710 of the transcriptional activity of the wntD promoter enables a quantitative analysis of its 711 dynamics. Embryos express His:RFP which marks nuclei in red and the transcriptional activity 712 is indicated by the green signal. (B-F) A constant reduction in the number of nuclei expressing 713 wntD is observed from NC12 onwards, such that by NC 14 no transcription is observed. Since 714 the wntD promoter responds to the level of $\mathrm{Dl}$, we assume that this reduction reflects the 715 attenuating activity of WntD.

716 Figure 6. Graded accumulation of T48 transcripts is driven by the dynamics of DI717 nuclear entry. (A) Time lapse images from the beginning of NC 14, showing the dynamics of 718 the onset of $T 48$ transcription in a wt embryo, followed by T48::MS2. Nuclei displaying 719 transcription were highlighted in yellow. Arrows mark the edges of the domain at each time 720 point. (B) Quantification of the data in (A). Times are color-coded according to (A), Y axis 721 represents the normalized number of transcription start sites (TSs) along the A-P axis, $\mathrm{X}$ axis 722 represents the position along the D-V axis. (C) The intensity of T48::MS2 signal for nuclei 723 across the $\mathrm{D}-\mathrm{V}$ axis at $33 \mathrm{~min}$. Once T48 transcription is initiated, it progresses at comparable 724 rates in all nuclei. (D,E) Single molecule FISH for T48 showing the intensity of transcription 725 and the number of active nuclei across the region expressing T48. Nuclei are marked by DAPI 
726 (blue) and T48 probe in red. Once transcription is initiated it proceeds at a similar rate by all 727 nuclei, and the number of nuclei expressing T48 in ventral and ventro-lateral regions is 728 comparable. (F) The ventral T48 expression domain, arrows mark TSs. (G) Quantification of 729 smFISH data: Number of T48 mRNA molecules in black bars, and TSs intensity corresponding 730 to transcription rate in blue diamonds.

731 Figure 7. Dynamics of Spz shuttling establishes pattern. The shuttling mechanism operates 732 within a wide domain D-V predefined by Pipe expression during oogenesis. Initially, a double733 peak distribution of the Spz morphogen in the peri-vitelline space is observed. In $w t$ embryos 734 shuttling operates efficiently to concentrate the Spz morphogen to a single sharp peak. The 735 dynamics of the Dl gradient are utilized by the embryo to induce graded accumulation of T48 736 transcripts, which facilitate recruitment of RhoGEF2 and ultimately Rho1 and actomyosin, to 737 drive apical constriction of invaginating mesodermal cells. Loss of WntD renders shuttling less 738 efficient, leading to a flattened peak of morphogen distribution. This may result in impaired 739 distribution of T48 transcripts and therefore in a broader gastrulation furrow. 


\section{$745 \quad$ Figure 2}
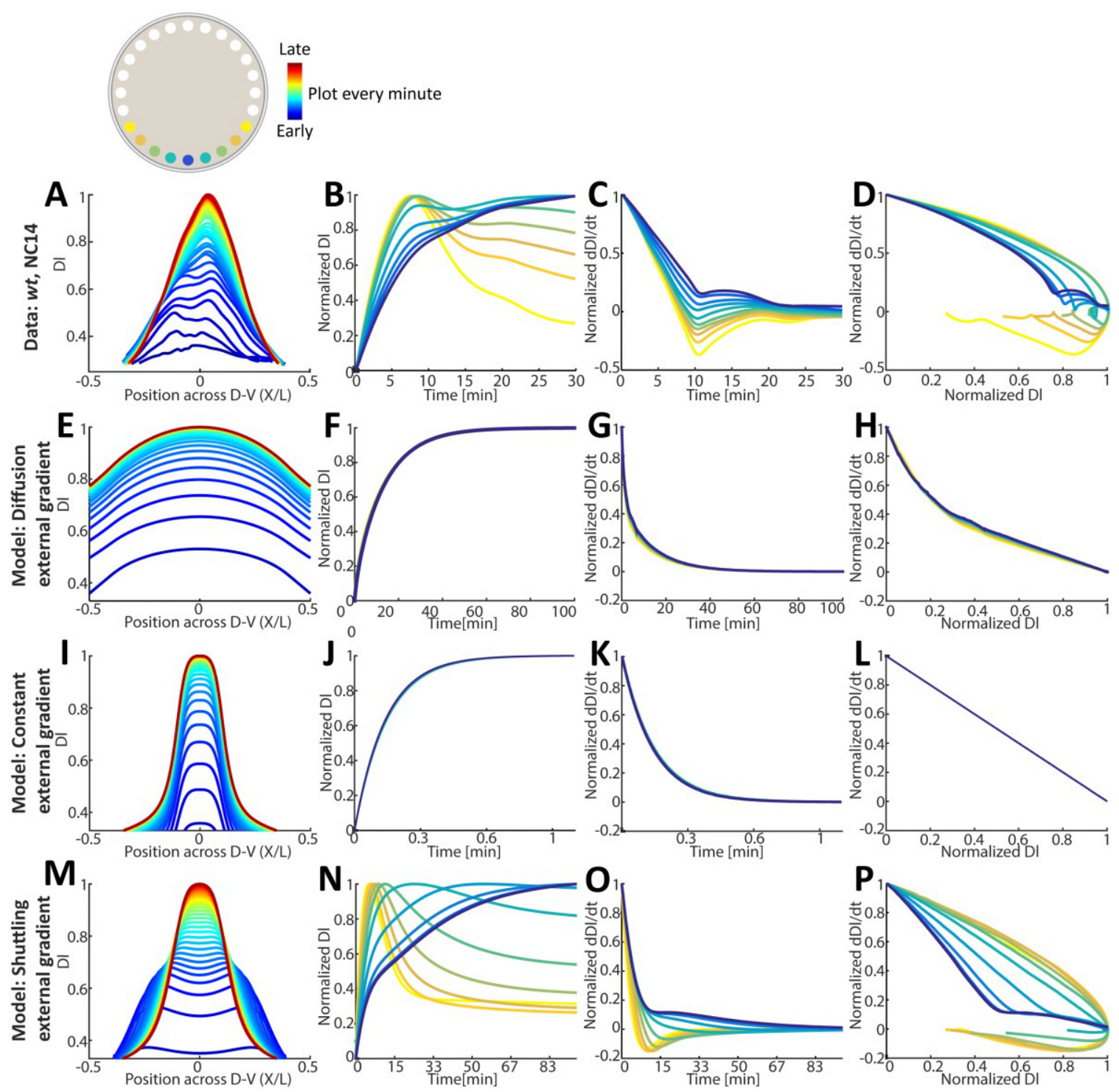


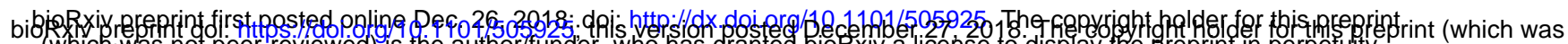

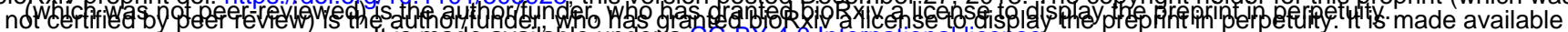

Figure 3
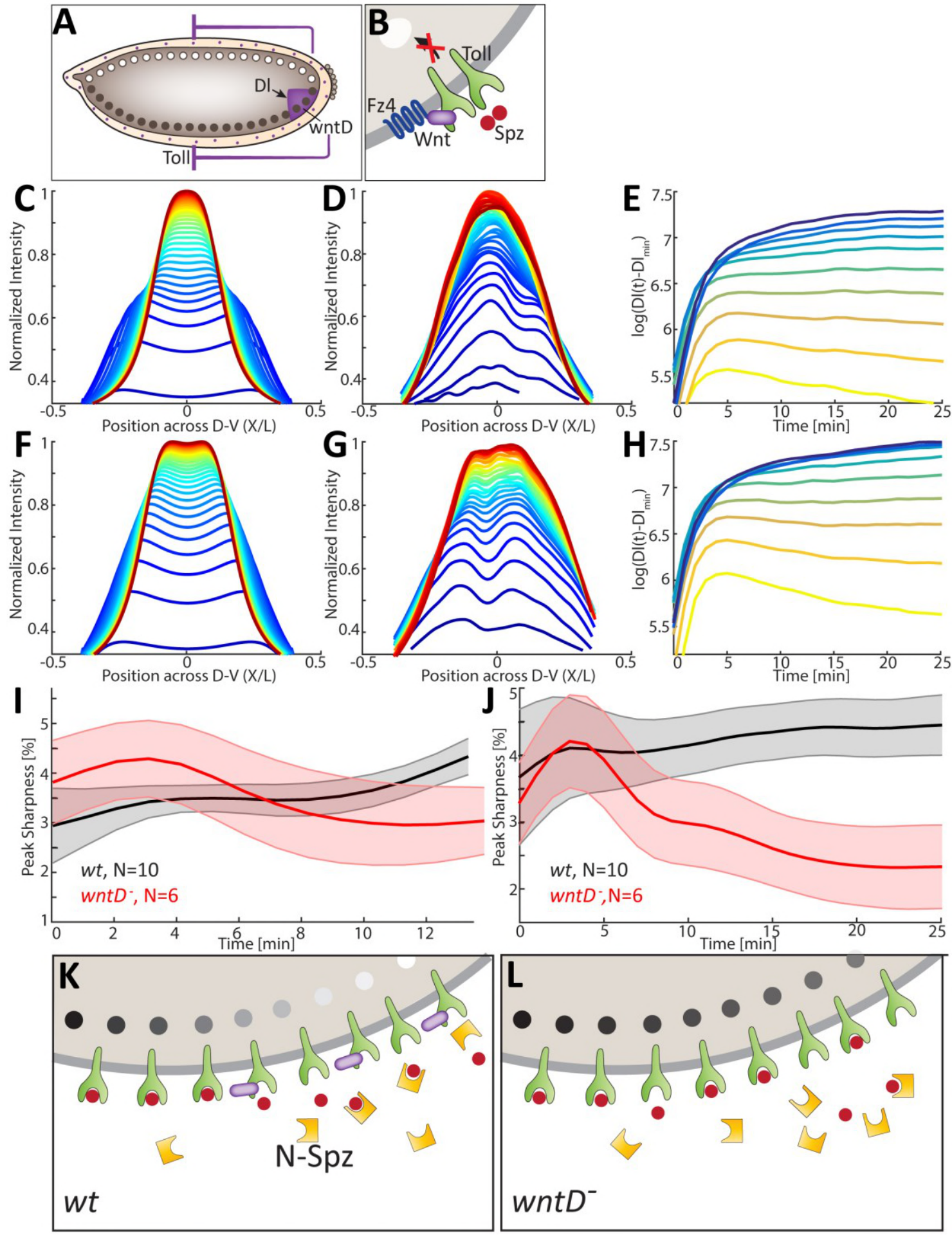

749 


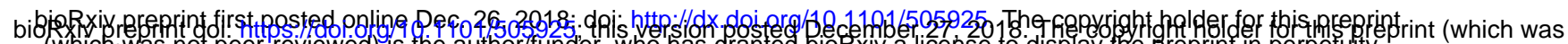

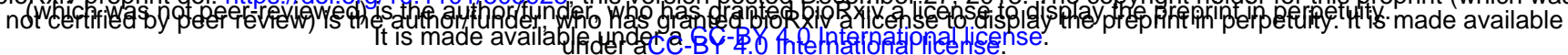

755 Figure 5

A

\begin{tabular}{lcccc}
\hline & 2000 & 4000 & 6000 & 8000 \\
\hline \multirow{2}{*}{ whtD Regulatory } & MS2 X 24 & & fhos-RH & \\
\hline
\end{tabular}
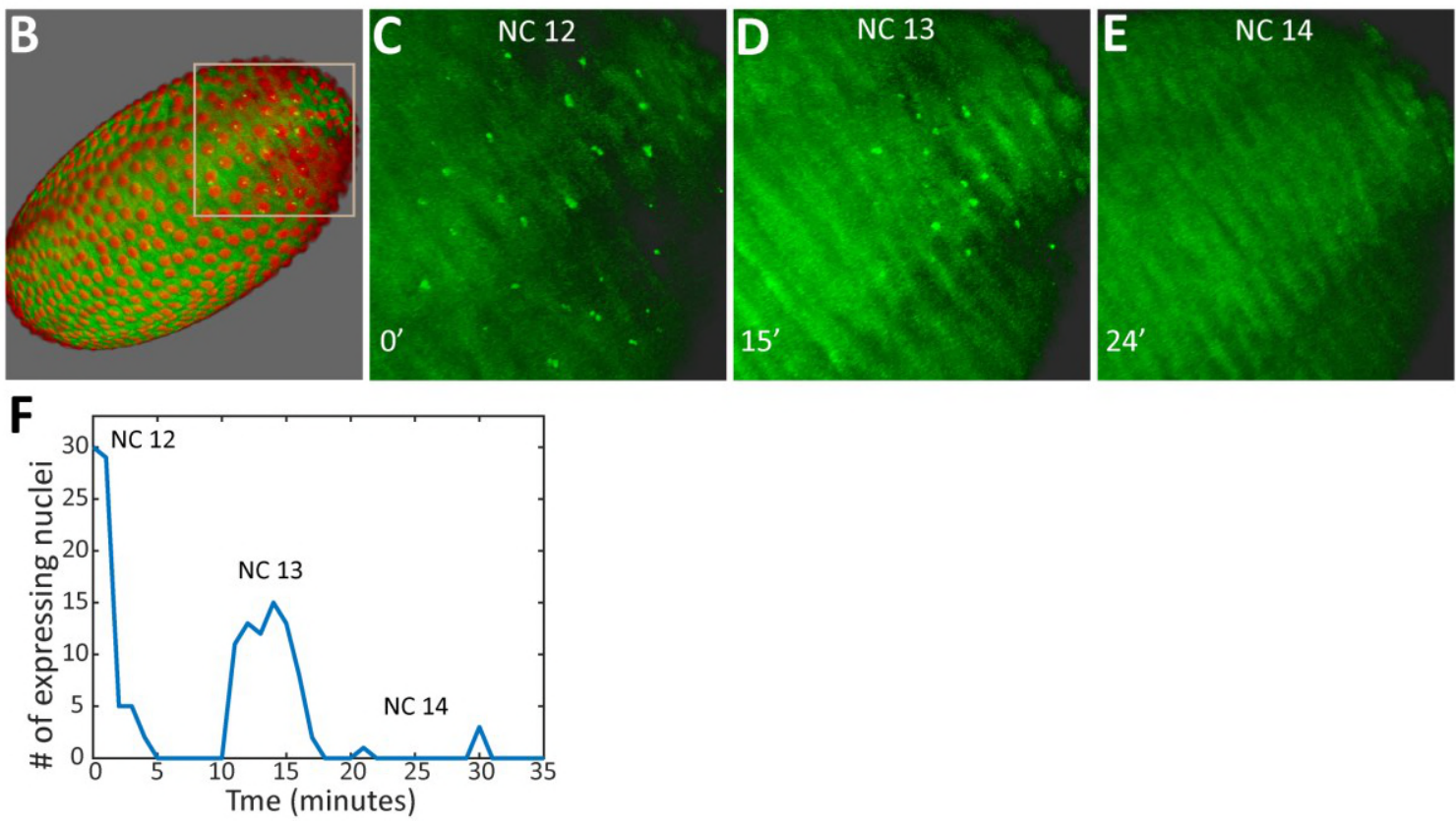

756

757 


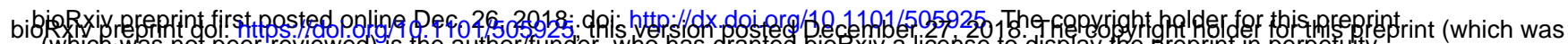

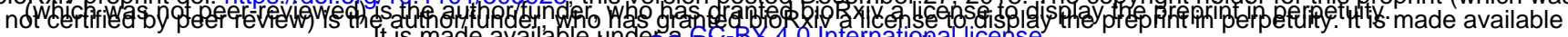

$761 \quad$ Figure 7

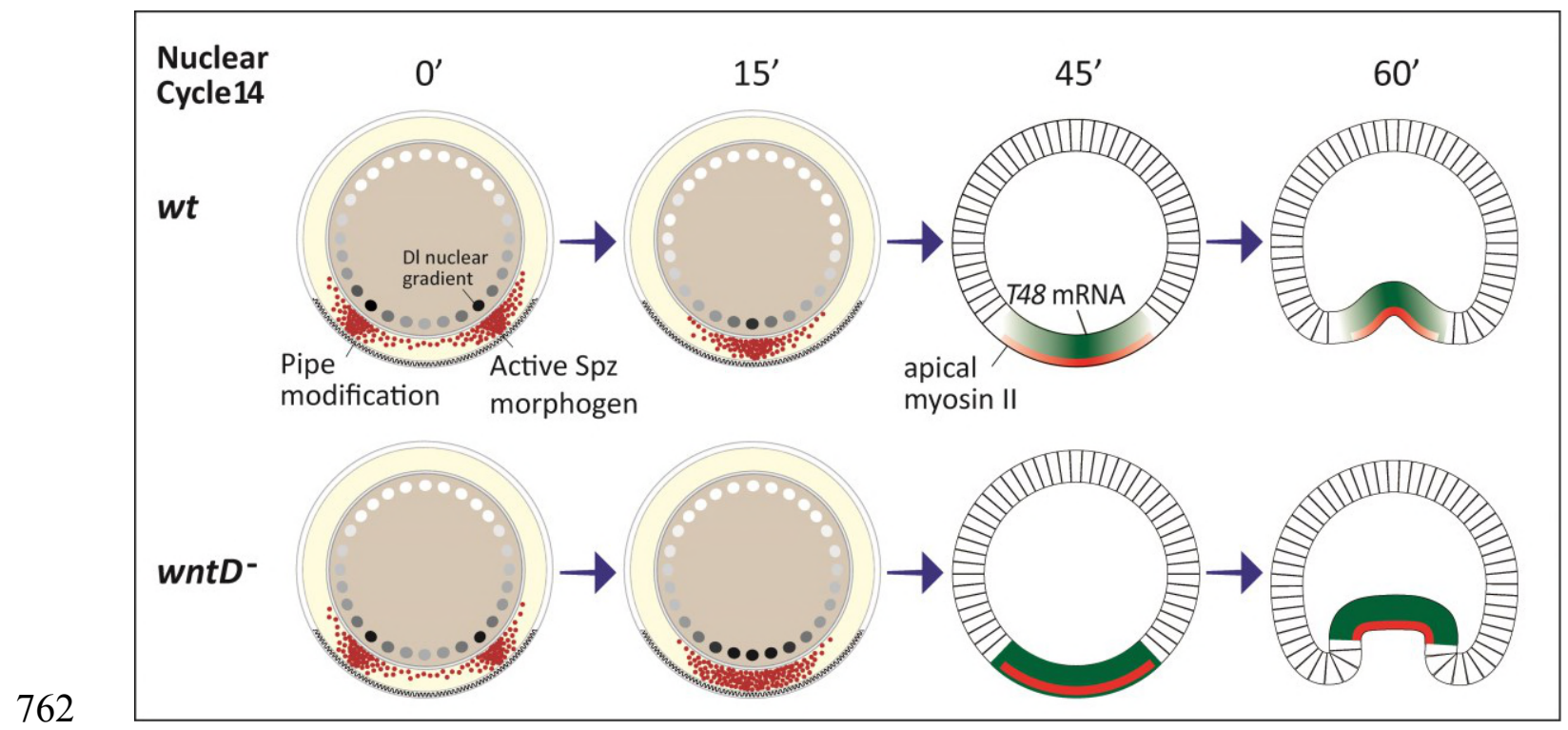

763 


\section{Dynamics of Spaetzle morphogen shuttling in the Drosophila \\ 765 embryo shapes pattern}

\section{Supplemental Information}

767 Contents

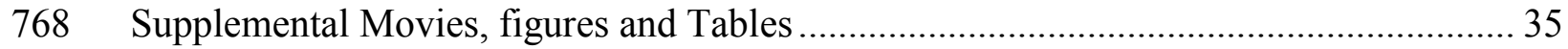

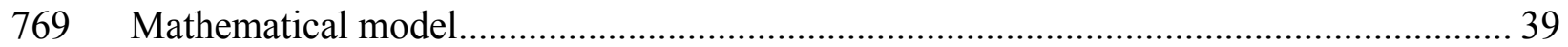

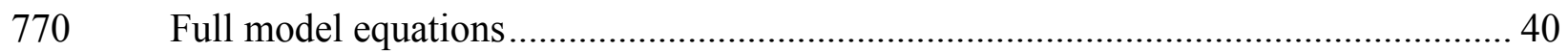

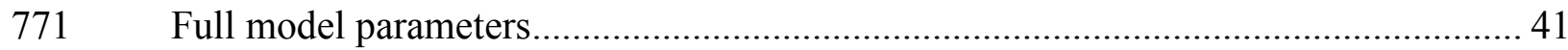

$772 \quad$ Constant external gradient model ............................................................................. 41

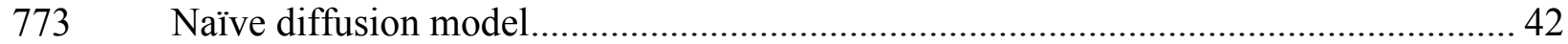

774 Shuttling parameters effect on double peak prominence .............................................. 42

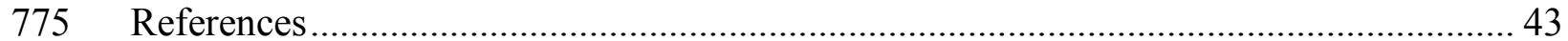

776

777 


\section{Supplemental Movies, figures and Tables}

Movie 1 - A Light Sheet time-lapse movie following the dynamics of endogenously expressed Dl-GFP in the entire embryo. The nuclear Dl gradient can be seen in nuclei at the ventral side (bottom) already at NC 12, it is lost during nuclear divisions and is re-generated at the onset of each nuclear cycle.

Movie 2 - A frame by frame 2D projection of movie 1 done using the ImSAnE tool (Heemskerk and Streichan, 2015). D1-GFP appears in grey.

Movie 3 - Time lapse of Dl-GFP intensity data for the area inside the dashed frame in Figure 1C. Each circular marker in the movie shows raw, non smoothed Dl-GFP intensity in a single nucleus. Nuclei were segmented from the corresponding frames of movie 2 (See Methods).
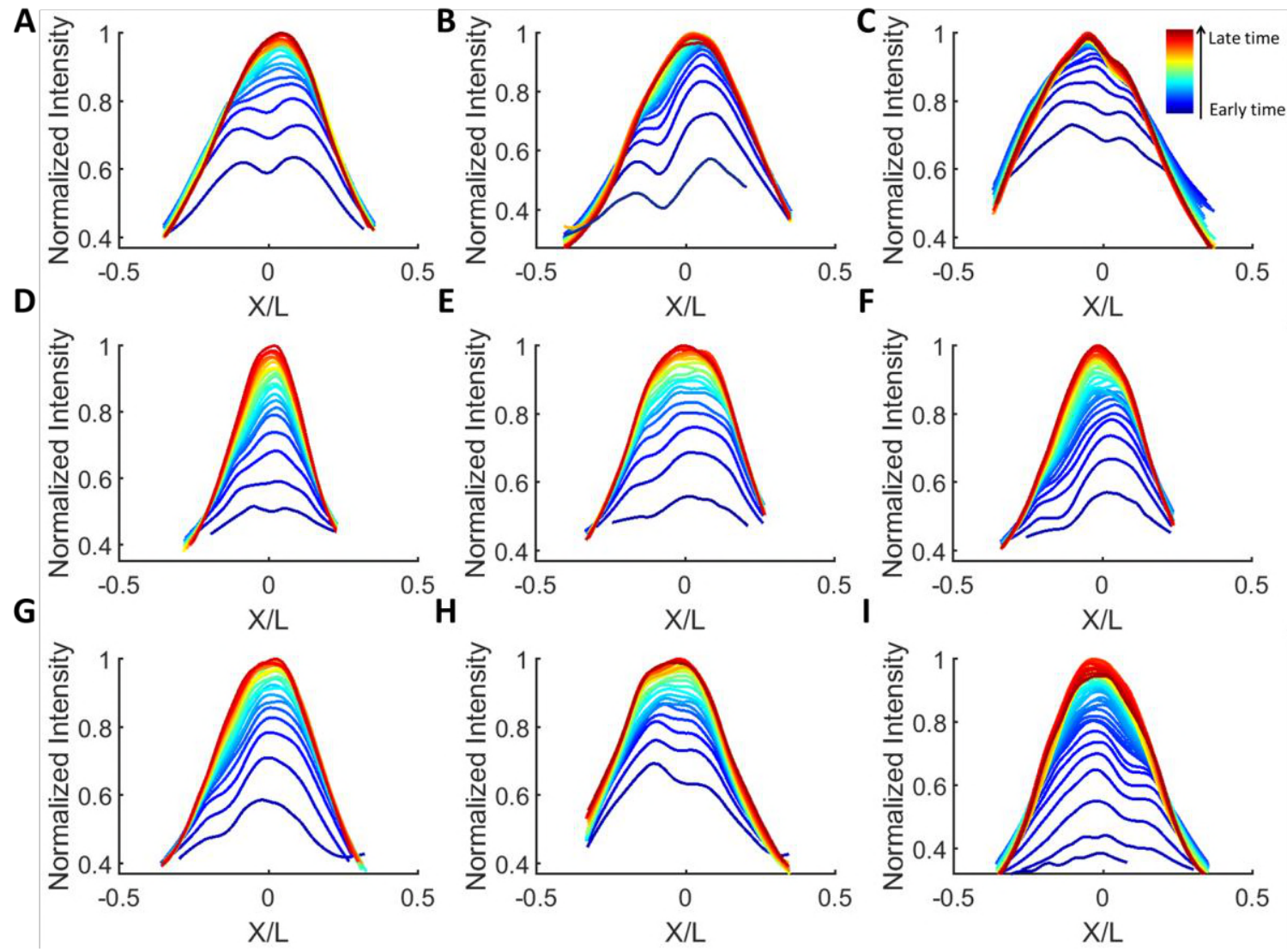

790

Figure S1 - Dl temporal dynamics during NC14 in wt embryos. Dl-GFP intensity, plotted as function of relative location along the DV axis, relative location axis $\mathrm{x} / \mathrm{L}$ is defined as location divided by embryo circumference (See Methods). A curve is shown for each time point during NC14 for $9 w t$ embryos. Each Dl-GFP intensity curve was smoothened and normalized by the maximal value attained during NC14 (See Methods). Time points are 1.5 minutes apart for (A-H) and 1 minute apart for I, going from earliest time points in blue to the latest in red. All embryos exhibit shuttling signatures: lateral overshoots and converging double peaks. 
A

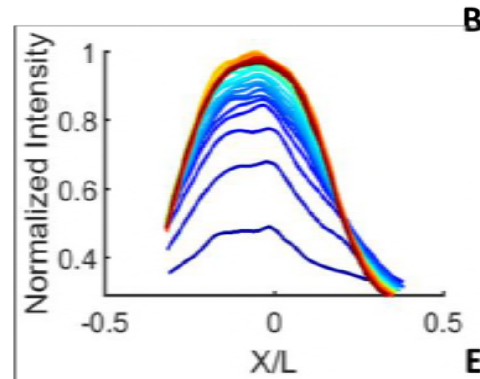

D

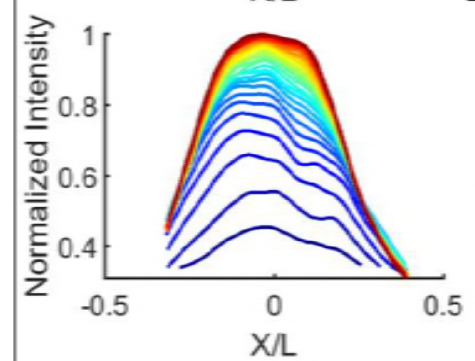

B

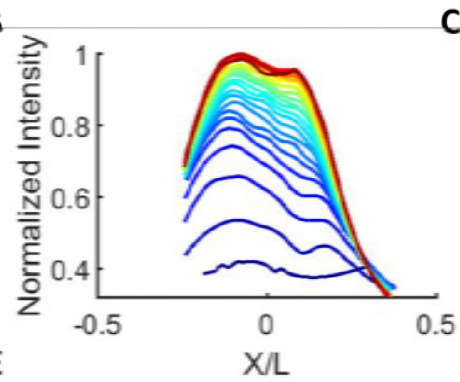

E

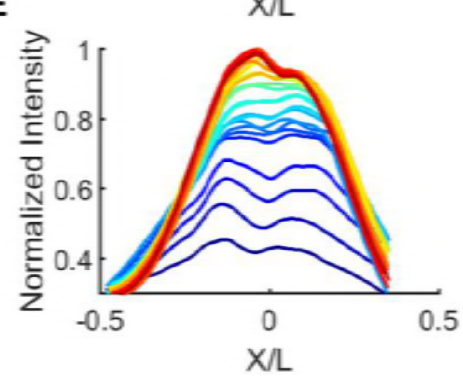

C

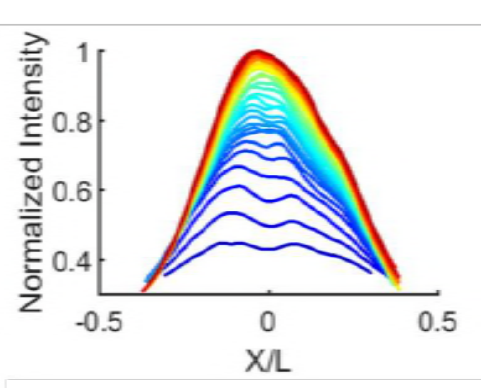

$\left\lceil\begin{array}{l}\text { Late time } \\ \text { Early time }\end{array}\right.$
797

798

799

800

801

802

Figure S2 - Dl temporal dynamics during NC14 in wntD-/- embryos. D1-GFP intensity, plotted as function of relative location along the DV axis, relative location axis $\mathrm{x} / \mathrm{L}$ is defined as location divided by embryo circumference (See Methods). A curve is shown for each time point during NC14 for 5 wntD-/- embryos. Each Dl-GFP intensity curve was smoothened and normalized by the maximal value attained during NC14 (See Methods). Time points are 1.5 minutes apart for, going from earliest time points in blue to the latest in red. All embryos exhibit shuttling signatures: lateral overshoots and double peaks flattening over time.

A

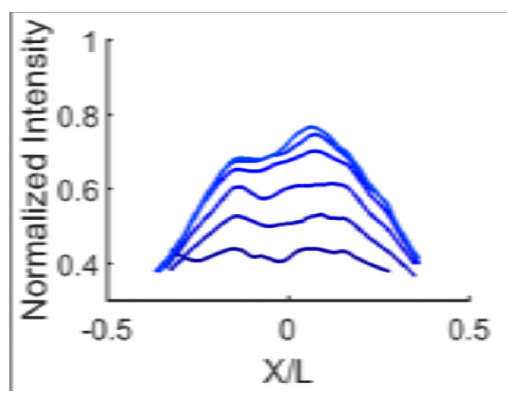

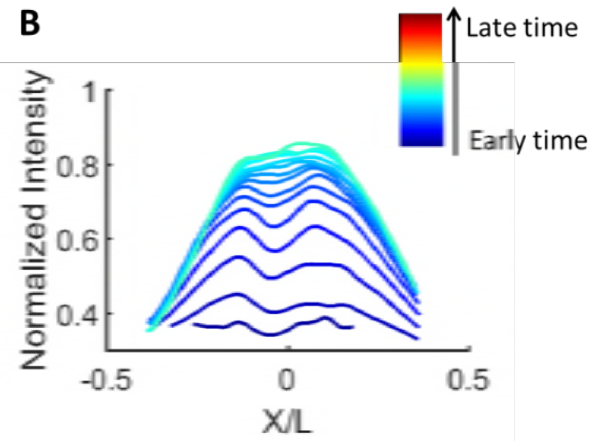

804 Figure S3 - Dl temporal dynamics during NC12-13 in a wntD-/- embryo. Dl-GFP intensity, plotted as function of relative 805 location along the DV axis, relative location axis $\mathrm{x} / \mathrm{L}$ is defined as location divided by embryo circumference (See Methods). 806 A curve is shown for each time point during NC12 (A) and NC13 (B) for the wntD-/- embryo presented in Figure 3E. Each 807 Dl-GFP intensity curve was smoothened and normalized by the maximal value attained during NC14 (See Methods, Figure 808 3E). Time points are 1 minute apart for, going from earliest time points in blue to the latest in red. 
Table S1 : Parameters table for full model, WT values

\begin{tabular}{|c|c|c|c|c|c|c|}
\hline$\#$ & Parameter & $\begin{array}{l}\text { Units } \\
\text { type }\end{array}$ & Meaning & $\begin{array}{l}\text { Value } \\
\text { in a.u }\end{array}$ & Units & $\begin{array}{l}\text { Value in } \\
\text { units }\end{array}$ \\
\hline 1 & $K_{\text {rec }}$ & $t^{-1}$ & Toll receptor recycling rate & 0.07 & $\sec ^{-1}$ & 0.007 \\
\hline 2 & $K_{\text {end }}$ & $t^{-1}$ & Toll receptor endocytosis rate & 1 & $\sec ^{-1}$ & 0.1 \\
\hline 3 & $\lambda$ & $t^{-1}$ & NCSpz* cleavage rate & 4.2 & $\sec ^{-1}$ & 0.42 \\
\hline 4 & $\alpha_{N}$ & $t^{-1}$ & NSpz degradation rate & 0.0011 & $\sec ^{-1}$ & 0.00011 \\
\hline 5 & $\alpha_{c}$ & $t^{-1}$ & CSpz degradation rate & 0.0014 & $\sec ^{-1}$ & 0.00014 \\
\hline 6 & $\alpha_{N c}$ & $t^{-1}$ & NCSpz degradation rate & 0.0016 & $\sec ^{-1}$ & 0.00016 \\
\hline 7 & $K_{\text {on }, c}$ & $t^{-1} C$ & CSpz binding to Toll & 0.3 & $\mu M \sec ^{-1}$ & 0.0013 \\
\hline 8 & $K_{o f f, c}$ & $t^{-1} C$ & CSpz un-binding from Toll & 1.12 & $\mu M \sec ^{-1}$ & 0.0049 \\
\hline 9 & $K_{\text {on }}$ & $t^{-1} C$ & NCSpz binding to Toll & 0.008 & $\mu M \sec ^{-1}$ & $3.2 \mathrm{e}-5$ \\
\hline 10 & $K_{o f f}$ & $t^{-1} C$ & NCSpz un-binding from Toll & 0.1 & $\mu M \sec ^{-1}$ & $4.33 e-04$ \\
\hline 11 & $\eta_{0}$ & $t^{-1} C$ & NCSpz activation rate & 0.22 & $\mu M \sec ^{-1}$ & $9.68 \mathrm{e}-4$ \\
\hline 12 & $K_{\text {bind }}$ & $t^{-1} C$ & CSpz binding rate to $\mathrm{NSpz}$ & 7 & $\mu M \sec ^{-1}$ & 0.0308 \\
\hline 13 & $K_{\text {split }}$ & $t^{-1}$ & $\begin{array}{l}\text { NC-Tl splitting rate to C-Tl } \\
\text { and } 2^{*} \mathrm{~N}-\mathrm{Spz}, \text { on Toll }\end{array}$ & 10 & $\sec ^{-1}$ & 1 \\
\hline 14 & $D_{N C}$ & $x^{2} t^{-1}$ & NCSpz diffusion coefficient & 0.1186 & $\mu m^{2} \sec ^{-1}$ & 118.6 \\
\hline 15 & $D_{N}$ & $x^{2} t^{-1}$ & NSpz diffusion coefficient & 0.0055 & $\mu m^{2} \sec ^{-1}$ & 55 \\
\hline 16 & $D_{N C *}$ & $x^{2} t^{-1}$ & NCSpz* diffusion coefficient & 0.745 & $\mu m^{2} \sec ^{-1}$ & 745 \\
\hline 17 & $T_{\text {tot }}$ & $C$ & Total Toll level & 5.625 & $\mu M$ & 0.25 \\
\hline 18 & $D_{W}$ & $x^{2} t^{-1}$ & WntD diffusion coefficient & 0.5 & $\mu m^{2} \sec ^{-1}$ & 500 \\
\hline 19 & $\alpha_{w}$ & $t^{-1}$ & WntD degradation rate & 0.001 & $\sec ^{-1}$ & $1.4 \mathrm{e}-5$ \\
\hline 20 & $\beta_{w}$ & $t^{-1} C$ & WntD production rate & 0.1 & $\mu \mathrm{Msec}^{-1}$ & $4.4 \mathrm{e}-4$ \\
\hline 21 & $K_{w}$ & $C^{n_{w}}$ & $\begin{array}{l}K_{D} \text { of WntD production as a } \\
\text { function of signaling }\end{array}$ & $9 e-10$ & $\mu M^{n_{w}}$ & $4.7 e-30$ \\
\hline 22 & $n_{w}$ & - & $\begin{array}{l}\text { Hill coefficient of WntD } \\
\text { production as a function of } \\
\text { signaling }\end{array}$ & 15 & - & 15 \\
\hline 23 & $L$ & $x$ & Length of tissue & 2 & $\mu m$ & 250 \\
\hline
\end{tabular}




\begin{tabular}{|c|c|c|c|c|c|c|}
\hline 24 & $X_{\text {pipe }}$ & $\%$ & $\begin{array}{l}\% \text { of L below which a point } \\
\vec{x}=(x, y, z) \in \text { pipe domain }\end{array}$ & 40 & $\%$ & 40 \\
\hline 25 & $X_{W n t D}$ & $\%$ & $\begin{array}{l}\text { \% of } \mathrm{L} \text { above which a point } \\
\vec{x}=(x, y, z) \in \\
\text { WntD producing domain }\end{array}$ & 25 & $\%$ & 25 \\
\hline 26 & $W_{\text {lower }}$ & $\%$ & $\begin{array}{l}\text { Maximal lowering of } \mathrm{Tl} \\
\text { property by WntD }\end{array}$ & 60 & $\%$ & 60 \\
\hline 27 & $W_{T r}$ & $C^{n_{\text {lower }}}$ & $\begin{array}{l}K_{D} \text { of WntD lowering } \\
\text { receptor property }\end{array}$ & 5 & $\mu M^{n_{\text {lower }}}$ & 0.01 \\
\hline 28 & $n_{\text {lower }}$ & - & $\begin{array}{l}\text { Hill coefficient of WntD } \\
\text { lowering receptor property }\end{array}$ & 2 & - & 2 \\
\hline 29 & $T$ & $t$ & $\begin{array}{l}\# \text { of discrete time points the } \\
\text { simulation was run }\end{array}$ & 600 & sec & $\begin{array}{l}6000 \\
(\sim 1.5 \\
\text { hours })\end{array}$ \\
\hline 30 & $K_{D l, i n}$ & $t^{-1} C$ & $\begin{array}{l}\text { Rate of Dl entry into the } \\
\text { nucleus }\end{array}$ & 0.07 & $\mu M \sec ^{-1}$ & $2.8 \mathrm{e}-04$ \\
\hline 31 & $K_{D l, \text { out }}$ & $t^{-1} C$ & $\begin{array}{l}\text { Rate of D1 exit from the } \\
\text { nucleus }\end{array}$ & 2 & $\mu M \sec ^{-1}$ & 0.008 \\
\hline 32 & $D l_{0}$ & $C$ & $\begin{array}{l}\text { Initial Dl concentration in the } \\
\text { nuclei }\end{array}$ & $1 \mathrm{e}-4$ & $\mu M$ & $4.4 \mathrm{e}-06$ \\
\hline 33 & $D l_{t o t}$ & $C$ & Total amount of Dl & 5 & $\mu M$ & 0.22 \\
\hline
\end{tabular}

811 


\section{Mathematical model}

815 Our full model describes how the Spz gradient is formed by shuttling, induces the nuclear

816 localization of Dl and the negative feedback between WntD and Dorsal which maintains the 817 gradients robustness (Rahimi et al., 2016). To this end, we extend the model from our previous 818 paper (Rahimi et al., 2016) to include the nuclear localization of Dorsal. Also, we used a

819 different mechanism by which WntD contracts the Spz gradient: instead of competing with the

820 ligand for binding the Toll receptor, we assume here that WntD "crowds" the Toll receptors

821 immediate environment by binding its own receptor Frizzled4 and limiting the access of Spz to

822 Toll. This increases the chances of free ligand binding the shuttling molecule instead of the 823 receptor and therefore enhances shuttling. This "crowding" of Toll has an additional affect:

824 stabilizing the ligand which succeeded in binding, which also makes shuttling more efficient.

825 The governing set of reaction-diffusion equations of our model is given below: eqn. 1 defines

826 the temporal dynamics of freely diffusing WntD. The terms of the equation by order of 827 appearance describe: WntD diffusion, WntD degradation, WntD production which depends on 828 nuclear Dorsal. This last term is the induction part of $\mathrm{InC}$ as WntD production is positively 829 regulated by signaling. The WntD producing zone is restricted and is defined in the embryo by 830 the Torso signaling border. In the simulations we define this zone using the model parameter $831 X_{W n t D}$ : WntD production is only allowed for points posterior to $X_{W n t D}$. Eqn. 2 defines the 832 nonlinear saturating function $R_{w}(\vec{x}, t)$ through which WntD changes the properties of the Toll

833 receptor: the rate at which ligands bind and unbind from it. Eqn. 3 defines the temporal 834 dynamics of free Toll receptors. This equation introduces the following constraint: the total 835 amount of Toll receptors (free, bound by ligand and endocytosed) is constant and equals $T_{\text {tot }}$.

836 Equations 4-10 are the self-organized shuttling model (SOSH) equations as appear in (Haskel837 Ittah et al., 2012), we'll review SOSH and the equations briefly. The SOSH mechanism 838 depends on the versatility of the Spz protein. The separate inhibitor domain N-Spz and 839 activating region of Spz, C-Spz, generated after cleavage of the NC-Spz precursor (eqn. 4), can

840 interact with each other in three different modes. These modes facilitate a process of "self841 organized shuttling", where the active ligand C-Spz is shuttled and concentrated at the ventral842 most region giving rise to the sharp activation gradient of Toll. Equations 5-7 describe the 843 shuttling of the active C-Spz ligand (which cannot diffuse on its own) by the N-Spz inhibitor 844 when bound together as the NC-Spz* complex. Signaling occurs when C-Spz bound to Toll 
845 undergoes endocytosis (eqns. 5,8,10). Toll receptors undergo recycling back to the membrane

846 after endocytosis and the total concentration of Toll is constant (eqns. 10,3 respectively). The

847 C-Spz ligand and N-Spz inhibitor are products of NC-Spz complex separation when bound to

848 the Toll receptor (eqn. 4,9). NC-Spz is also capable of inducing Toll endocytosis when binding

849 it and thus contributing to signaling (eqn. 9) but signaling through NC-Spz happens at a much

850 lower rate than C-Spz mediated signaling. Equations 11-12 describe the induction of Dorsal

851 nuclear localization by Toll signaling. Eqn. 11 introduces the following constraint: the total

852 amount of Dorsal (Nuclear- $D l_{\text {in }}$ and cytoplasmic- $D l_{\text {out }}$ ) is constant and equals $D l_{\text {tot }}$. The

853 meaning of the different parameters and their units are summarized in Table S1. This set of

854 equations was solved numerically in 1D using a standard MATLAB PDE solver.

\section{Full model equations}

856

(1) $\frac{\partial[W]}{\partial t}=D_{W} \nabla^{2}[W]-\alpha_{w}[W]+\beta(\vec{x}) \frac{\left[D l_{\text {in }}\right]^{n_{W}}}{K_{w}+\left[D l_{i n}\right]^{n_{W}}}$

$$
\beta(\vec{x})=\left\{\begin{array}{lr}
\text { if } x>X_{W n t D} \rightarrow \vec{x}=(x, y, z) \in \text { wntD expression zone, } & \beta_{w} \\
\text { else, } & 0
\end{array}\right.
$$

(2) $R_{w}(\vec{x}, t)=W_{\text {lower }}+\left(1-W_{\text {lower }}\right) \frac{W_{T r}}{W_{T r}+[W(\vec{x}, t)]^{n} \text { lower }}$

859

(3) $T_{\text {tot }}=[T l]+[C S p z-T l]+[N C S p z-T l]+\left[T l_{\text {end }}\right] \rightarrow$

861

$$
[T l]_{(\vec{x}, t)}=T_{t o t}-[C S p z-T l]-[N C S p z-T l]+\left[T l_{\text {end }}\right]
$$

862

(4) $\frac{\partial[N C S p z]}{\partial t}=$

$\left.D_{N C} \nabla^{2}[N C S p z]-\alpha_{N C}[N C S p z]+\eta(\vec{x})-R_{w} K_{o n}[T l][N C S p z]+R_{w} K_{o f f}[N C S p z-T l]\right)$

$$
\eta(\vec{x})=\left\{\begin{array}{lr}
\text { if } \vec{x}=(x, y, z) \in \text { pipe domain, } & \eta_{0} \\
\text { else, } & 0
\end{array}\right.
$$

863

(5) $\frac{\partial[\operatorname{csp} z]}{\partial t}=$

$864-\alpha_{C}[\mathrm{CSpz}]-R_{w} K_{o n, c}[T l][C S p z]+R_{w} K_{o f f, c}[\mathrm{CSpz}-T l]-K_{\text {bind }}[\mathrm{NSpz}][\mathrm{CSp} z]+$

$K_{\text {Split }}[N C S p Z-T l]+\frac{\lambda \eta(\vec{x})}{\eta_{0}}[N C S p Z *]$

866

(6) $\frac{\partial[N S p z]}{\partial t}=D_{N C} \nabla^{2}[N S p z]-\alpha_{N C}[N S p z]-K_{b i n d}[N S p z][C S p z]+2 K_{S p l i t}[N C S p z-T l]$

867

$$
\text { (7) } \frac{\partial[N C S p Z *]}{\partial t}=D_{N C *} \nabla^{2}[N C S p z *]-\frac{\lambda \eta(\vec{x})}{\eta_{0}}[N C S p z *]+K_{b i n d}[N S p z][C S p z]
$$


(8) $\frac{\partial[\operatorname{CSp} z-T l]}{\partial t}=R_{w}[T l][C S p z]-R_{w} K_{o f f, c}[\operatorname{CSpz}-T l]+K_{s p l i t}[N C S p z-T l]-$

869

$$
K_{\text {end }}[\mathrm{CSpz}-\mathrm{Tl}]
$$

870

$$
\begin{gathered}
\text { (9) } \frac{\partial[N C S p z-T l]}{\partial t}=R_{w} K_{o n}[T l][N C S p z]-\left(R_{w} K_{o f f}+K_{\text {split }}+K_{\text {end }}\right)[N C S p z-T l] \\
(\mathbf{1 0}) \frac{\partial\left[T l_{\text {end }}\right]}{\partial t}=K_{\text {end }}([C S p z-T l]+[N C S p z-T l])-K_{\text {rec }}\left[T l_{\text {end }}\right] \\
(\mathbf{1 1})\left[D l_{T o t}\right]=\left[D l_{\text {in }}\right]+\left[D l_{\text {out }}\right] \\
\text { (12) } \frac{\partial\left[D l_{\text {in }}\right]}{\partial t}=K_{D l, \text { in }}\left[T l_{\text {end }}\right]\left[D l_{\text {out }}\right]-K_{D l, o u t}\left[D l_{\text {in }}\right]
\end{gathered}
$$

\section{Full model parameters}

873 When choosing parameters, we relied on our previous papers where SOSH (Haskel-Ittah et al., 874 2012) and InC (Rahimi et al., 2016) were previously analyzed. For the SOSH WT parameters 875 we in this work, we used the SOSH parameter values described in Table S5 of (Haskel-Ittah et 876 al., 2012) with slight alterations to better resemble our experimental data. Values for WntD 877 related parameters (diffusion, degradation, and production rates) were based on our previous 878 paper (Rahimi et al., 2016). The addition of Dorsal nuclear localization (eqns. 11-12) 879 introduced new dorsal related parameters, which were selected to resemble similar parameters

880 in the model (for example, the total concentration of Dorsal is similar to that of Toll). For the 881 simulation of WntD mutants, model equations were solved with the WntD production rate set 882 to zero.

\section{Constant external gradient model}

884 In order to simulate only Dorsal nuclear localization induced by a constant Toll signaling 885 profile, the following set of equations was solved:

$$
\begin{gathered}
\text { (13) } \frac{\partial\left[T l_{\text {end }}\right]}{\partial t}=0, \quad\left[T l_{\text {end }}\right]=T l_{\text {end }}^{\text {final }}(X) \\
(\mathbf{1 4})\left[D l_{T o t}\right]=\left[D l_{\text {in }}\right]+\left[D l_{\text {out }}\right] \\
\text { (15) } \frac{\partial\left[D l_{\text {in }}\right]}{\partial t}=K_{D l, i n}\left[T l_{\text {end }}\right]\left[D l_{\text {out }}\right]-K_{D l, o u t}\left[D l_{\text {in }}\right]
\end{gathered}
$$


886 The constant external gradient, $\mathrm{Tl} l_{\text {end }}^{\text {final }}$, was selected to be the sharp Toll signaling profile

$887\left[T l_{\text {end }}\right]$ at the last time point for the simulation of the full model (eqns 1-12). Parameter values 888 according to Table S1.

\section{Naïve diffusion model}

890 This model includes a single ligand, $N C S p z$, which is produced throughout the pipe domain 891 (eqn. 17). The ligand diffuses and is degraded (eqn. 17). It binds the Toll receptor (eqns. 892 18,16), and induces Dorsal nuclear localization (eqns. 20-21). This model is described by the 893 following equations:

(16) $T_{\text {tot }}=[T l]++[N C S p z-T l]+\left[T l_{\text {end }}\right] \rightarrow$

895

896

$$
\text { (17) } \frac{\partial[N C S p z]}{\partial t}=D_{N C} \nabla^{2}[N C S p z]-\alpha_{N C}[N C S p z]+\eta(\vec{x})-K_{o n}[T l][N C S p z]+K_{o f f}[N C S p z-
$$
$T l])$

$$
\eta(\vec{x})=\left\{\begin{array}{lr}
\text { if } \vec{x}=(x, y, z) \in \text { pipe domain, } & \eta_{0} \\
\text { else }, & 0
\end{array}\right.
$$

$$
\begin{aligned}
\text { (18) } \frac{\partial[N C S p z-T l]}{\partial t}=K_{o n}[T l][N C S p z]-\left(K_{\text {off }}+K_{\text {end }}\right)[N C S p z-T l] \\
\text { (19) } \frac{\partial\left[T l_{\text {end }}\right]}{\partial t}=K_{\text {end }}([N C S p z-T l])-K_{\text {rec }}\left[T l_{\text {end }}\right] \\
(\mathbf{2 0})\left[D l_{T o t}\right]=\left[D l_{\text {in }}\right]+\left[D l_{\text {out }}\right] \\
\text { (21) } \frac{\partial\left[D l_{\text {in }}\right]}{\partial t}=K_{D l, \text { in }}\left[T l_{\text {end }}\right]\left[D l_{\text {out }}\right]-K_{D l, o u t}\left[D l_{\text {in }}\right]
\end{aligned}
$$

898 Parameter values according to Table S1. See further analytical and numerical analysis for the

899 sharpness and robustness of the gradient in this model in (Haskel-Ittah et al., 2012).

\section{Shuttling parameters effect on double peak prominence}

901 The prominence of the converging double peak feature in the shuttling model depends on

902 model parameters which control the mean path the shuttling complex travels ventrally 903 before being cleaved, relative to the length of the source (pipe domain). This mean path is 904 influenced by several parameters, mainly tissue absolute length $L$ (when maintaining the 905 source as $40 \%$ of absolute length), changing source length directly by assuming the pipe 906 domain is smaller or larger than $40 \%$ of the circumference, cleavage rate of the shuttling 907 complex $\lambda$, diffusion coefficient of the shuttling complex $D_{N C *}$. For a sufficiently large ratio 
908 between the mean path and $L$, no double peak will be observed. Lowering this ratio

909 increases the prominence of the double peak. For a sufficiently small ratio, the double

910 peak does not converge into a single peak since the shuttling complex cannot penetrate

911 all the way to the ventral most. We demonstrate this by solving the full model for our WT

912 parameter set (Table S1), and comparing to sets where these parameters are perturbed:

913 decreased 2 fold and increased 2 fold:

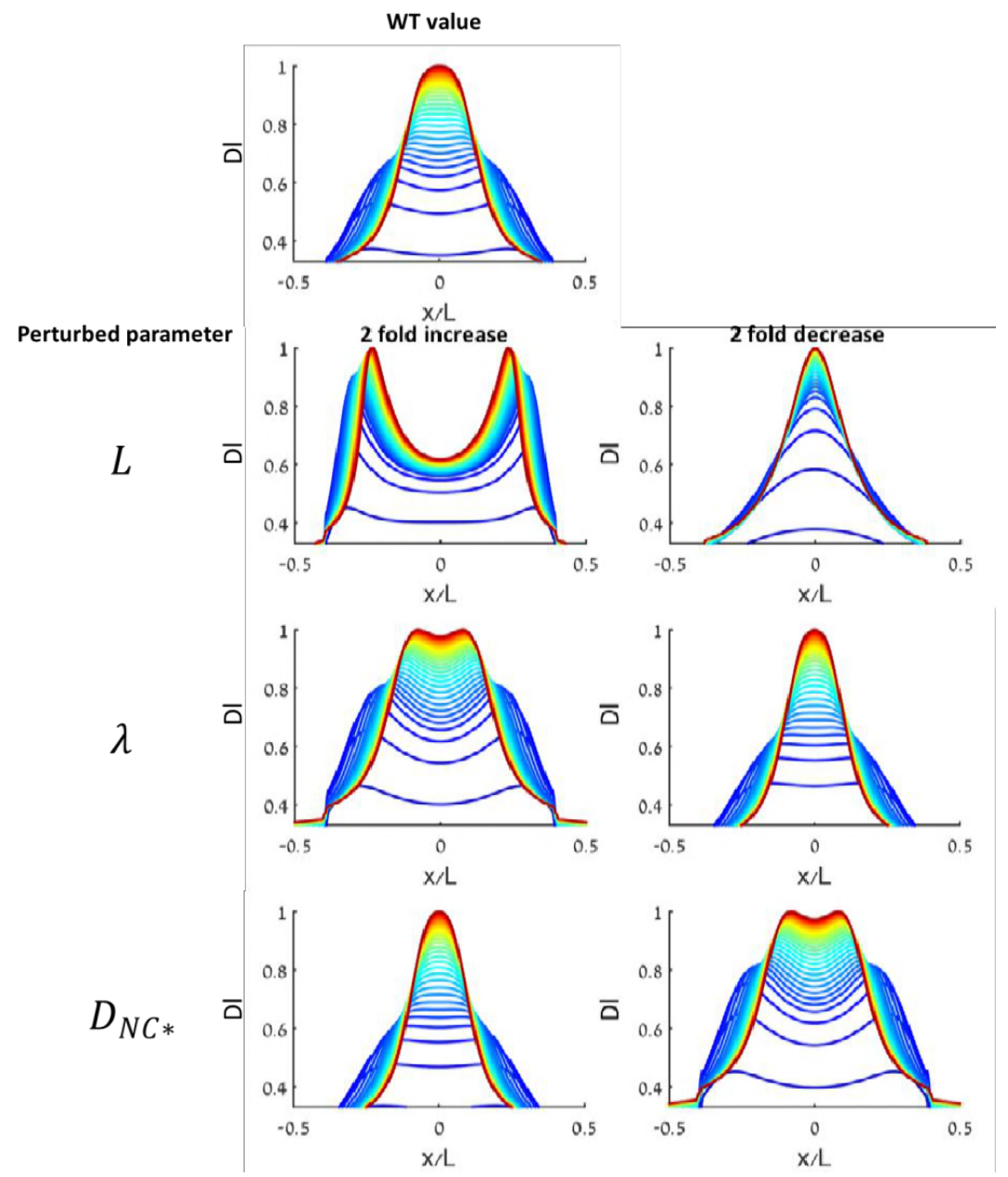

Model Supplement Figure 1 - Perturbations to shuttling complex parameters modulate double peak prominence.

\section{References}

917 Haskel-Ittah, M., Ben-Zvi, D., Branski-Arieli, M., Schejter, E.D., Shilo, B.Z., Barkai, N., 2012. 918 Self-organized shuttling: generating sharp dorsoventral polarity in the early Drosophila 919 embryo. Cell 150, 1016-1028.

920 Heemskerk, I., Streichan, S.J., 2015. Tissue cartography: compressing bio-image data by 921 dimensional reduction. Nat Methods 12, 1139-1142. 
922 Rahimi, N., Averbukh, I., Haskel-Ittah, M., Degani, N., Schejter, E.D., Barkai, N., Shilo, B.Z., 923 2016. A WntD-Dependent Integral Feedback Loop Attenuates Variability in Drosophila Toll 924 Signaling. Dev Cell 36, 401-414.

925 